\title{
Precise discussion of time-reversal asymmetries in B-meson decays
}

\section{Takuya Morozumi, Hideaki Okane and Hiroyuki Umeeda}

Graduate School of Science, Hiroshima University, Higashi-Hiroshima, Hiroshima 739-8526, Japan

Core of Research for the Energetic Universe, Hiroshima University,

Higashi-Hiroshima, Hiroshima 739-8526, Japan

E-mail: morozumi@hiroshima-u.ac.jp, hideaki-ookane@hiroshima-u.ac.jp, umeeda@theo.phys.sci.hiroshima-u.ac.jp

ABSTRACT: BaBar collaboration announced that they observed time reversal (T) asymmetry through $B$ meson system. In the experiment, time dependencies of two distinctive processes, $B_{-} \rightarrow \bar{B}^{0}$ and $\bar{B}^{0} \rightarrow B_{-}(-$expresses $\mathrm{CP}$ value $)$ are compared with each other. In our study, we examine event number difference of these two processes. In contrast to the BaBar asymmetry, the asymmetry of events number includes the overall normalization difference for rates. Time dependence of the asymmetry is more general and it includes terms absent in one used by BaBar collaboration. Both of the BaBar asymmetry and ours are naively thought to be T-odd since two processes compared are related with flipping time direction. We investigate the time reversal transformation property of our asymmetry. Using our notation, one can see that the asymmetry is not precisely a T-odd quantity, taking into account indirect $\mathrm{CP}$ and $\mathrm{CPT}$ violation of $\mathrm{K}$ meson systems. The effect of $\epsilon_{K}$ is extracted and gives rise to $\mathcal{O}\left(10^{-3}\right)$ contribution. The introduced parameters are invariant under rephasing of quarks so that the coefficients of our asymmetry are expressed as phase convention independent quantities. Some combinations of the asymmetry enable us to extract parameters for wrong sign decays of $B_{d}$ meson, CPT violation, etc. We also study the reason why the T-even terms are allowed to contribute to the asymmetry, and find that several conditions are needed for the asymmetry to be a T-odd quantity.

Keywords: B-Physics, CP violation, Kaon Physics

ArXiv EPRINT: 1411.2104 


\section{Contents}

1 Introduction 1

2 Formula for asymmetry of entangled systems 3

3 Parameter definitions in terms of flavor based state 4

4 Time dependent asymmetry including the overall constants 10

4.1 Extracting the parameters from the coefficients: general case 14

4.2 Extracting the parameters from the coefficients: CPT conserving limit 16

4.3 Extracting the parameters from the coefficients: case without wrong sign decay 17

$\begin{array}{lll}5 & \text { Conditions for authentic time reversed process } & 17\end{array}$

$\begin{array}{llr}6 & \text { Conclusion } & 20\end{array}$

$\begin{array}{ll}\text { A Coefficients of master formula } & 21\end{array}$

B Incoming mass eigenstates and outgoing mass eigenstates in B meson $\begin{array}{ll}\text { and } \mathrm{K} \text { meson system } & 22\end{array}$

C List of coefficients of time dependent decay rates for process (I) 23

D Expressions for $N_{R}, \Delta \mathcal{S}, \Delta \mathcal{C}, \Delta \sigma, \hat{\sigma}, \hat{\mathcal{S}}$ and $\hat{\mathcal{C}}$

E The relation among coefficients of the asymmetries for processes (I)-(IV) 25

$\begin{array}{lll}\text { F Calculation of equivalence conditions } & 26\end{array}$

\section{Introduction}

T-symmetry is fundamental symmetry in particle physics. T-transformation exchanges an initial state and final state, flipping the momentum and spin of particles. If some rate of a process deviates from the rate of time reversed one, it implies T-violation. T-violation is worth pursuit since it reflects the characterized feature of theory.

In EPR correlating B meson system, if one of a pair of B meson is tagged, another side of $\mathrm{B}$ meson is determined as orthogonal state with tagged side $\mathrm{B}$ meson. In refs. [1][3], a method to observe T-violation using B meson system is suggested. Their idea is based on the difference of the time dependencies for two distinctive processes, $B_{-} \rightarrow \bar{B}^{0}$ and $\bar{B}^{0} \rightarrow B_{-}$. In refs. [1]-[3], it is considered to be T-asymmetry since two distinctive processes are related with flipping time direction. Then, BaBar collaboration announced [4] 
that they measured non-zero asymmetry and this observation is direct demonstration of T-violation. A review for the BaBar asymmetry and the other $\mathrm{T}$ and $\mathrm{CPT}$ asymmetries is given in ref. [5].

However, their statement includes ambiguity since we cannot exactly identify B meson such as $\bar{B}^{0}$ or $B_{-}$. In the BaBar experiment, two methods to identify $\bar{B}^{0}$ and $B_{-}$are implemented. The first one is referred as flavor tagging which enables one to identify $\overline{B^{0}}$, using the semi-leptonic decay mode of B meson. Another one is referred as CP tagging which allows us to identify $B_{-}$with final state $\psi K_{S}$.

In ref. [6], it is pointed out that there exist subtleties in BaBar measurement. The main point in ref. [6] is that they consider a process and its authentically time reversed process. Note that the authentic time reversed process includes inverse decay such like $l^{-} X \rightarrow \bar{B}^{0}$ and $\psi K_{L} \rightarrow B^{0}$. Since an authentic time reversed process is experimentally hard to observe, they substitute another process which does not include inverse decays. Therefore, they derive the conditions that BaBar asymmetry is identical with a T-odd quantity, taking into account inverse decays. The derived conditions are (1) the absence of the wrong sign semi-leptonic B meson decays, (2) the absence of the wrong strangeness decays, (3) the absence of CPT violation of the strangeness changing decays. All the conditions are derived by assuming that $\psi K_{S}$ and $\psi K_{L}$ are exact CP eigenstates.

In this paper, we conduct model-independent analysis of an event number asymmetry. Our analysis is extension of the work [6], incorporating the difference of overall constants for the rates that form the asymmetry into calculation. We also include the effect of indirect $\mathrm{CP}$ and $\mathrm{CPT}$ violation of $\mathrm{K}$ meson system. Furthermore, the asymmetry is written in terms of the phase convention independent parameters and one can find contribution of $\epsilon_{K}$ explicitly. Some combinations of the coefficients enable one to constrain model-independent parameters. We also discuss the T-even parts of the asymmetry. One can find that the asymmetry is a T-odd quantity when several conditions are satisfied.

In section 2, we introduce the asymmetry of entangled B meson system. In section 3, we define parameters which are definitively T-odd or T-even and describe the relation between notation in [6] and ours. It is also shown that the parameters are phase convention independent quantities. The method to extract the effect of indirect $\mathrm{CP}$ violation in Kaon system is also considered in section 3 . In section 4 , we write the event number asymmetry in terms of the parameters defined in section 3 and show that the asymmetry consists of not only the T-odd part but also T-even part, using our notation. In sections 4.1-4.3, rather than discussing T-transformation property of the asymmetry, some methods to extract the parameters from the asymmetry are investigated. In section 5, we derive the conditions that T-even parts of the asymmetry vanish and examine the intuitive reason why these conditions are required. The conditions are categorized as two types. The first condition is in regards to B meson states that appear in the processes. Including the effect of indirect $\mathrm{CP}$ violation, we evaluate how the first condition is violated, in comparison with the result in [6]. The second condition is in regards to overall constant which forms the asymmetry. We find that the second condition is needed when one takes account of the difference of overall constant of the two rates. Section 6 is devoted to summary of our study. 


\section{Formula for asymmetry of entangled systems}

In ref. [6], a formula for the time-dependent decay rate of the entangled $B \bar{B}$ system is derived. When $f_{1}$ and $f_{2}$ denote the final states of a tagging side and a signal side, respectively, it is written as,

$$
\begin{aligned}
\Gamma_{\left(f_{1}\right) \perp, f_{2}}= & e^{-\Gamma\left(t_{1}+t_{2}\right)} N_{(1) \perp, 2}\left[\kappa_{(1) \perp, 2} \cosh (y \Gamma t)+\sigma_{(1) \perp, 2} \sinh (y \Gamma t)\right. \\
& \left.+\mathcal{C}_{(1) \perp, 2} \cos (x \Gamma t)+\mathcal{S}_{(1) \perp, 2} \sin (x \Gamma t)\right] \\
= & e^{-\Gamma\left(t_{1}+t_{2}\right)} N_{(1) \perp, 2} \kappa_{(1) \perp, 2}\left[\cosh (y \Gamma t)+\frac{\sigma_{(1) \perp, 2}}{\kappa_{(1) \perp, 2}} \sinh (y \Gamma t)\right. \\
& \left.+\frac{\mathcal{C}_{(1) \perp, 2}}{\kappa_{(1) \perp, 2}} \cos (x \Gamma t)+\frac{\mathcal{S}_{(1) \perp, 2}}{\kappa_{(1) \perp, 2}} \sin (x \Gamma t)\right]
\end{aligned}
$$

where

$$
\Gamma=\frac{\Gamma_{H}+\Gamma_{L}}{2}, \quad x=\frac{m_{H}-m_{L}}{\Gamma}, \quad y=\frac{\Gamma_{H}-\Gamma_{L}}{2 \Gamma} .
$$

The expressions for $N_{(1) \perp, 2}, \kappa_{(1) \perp, 2}, \sigma_{(1) \perp, 2}, \mathcal{C}_{(1) \perp, 2}$ and $\mathcal{S}_{(1) \perp, 2}$ are given in ref. [6]. For the sake of completeness, we record their expressions in eqs. (A.1)-(A.5). Hereafter, we evaluate an asymmetry including overall factor $N_{(1) \perp, 2} \kappa_{(1) \perp, 2}$ in eq. (2.1). One obtains a generic formula for the event number asymmetry of the two distinctive sets for final states; $\left(f_{1}, f_{2}\right)$ versus $\left(f_{3}, f_{4}\right)$ as,

$$
\begin{aligned}
A \equiv & \frac{\Gamma_{\left(f_{1}\right) \perp, f_{2}}-\Gamma_{\left(f_{3}\right) \perp, f_{4}}}{\Gamma_{\left(f_{1}\right) \perp, f_{2}}+\Gamma_{\left(f_{3}\right) \perp, f_{4}}} \\
= & \frac{\left(\frac{1}{\sqrt{N_{R}}}-\sqrt{N_{R}}\right) \cosh (y \Gamma t)+\Delta \sigma \sinh (y \Gamma t)+\Delta \mathcal{S} \sin (x \Gamma t)+\Delta \mathcal{C} \cos (x \Gamma t)}{\left(\frac{1}{\sqrt{N_{R}}}+\sqrt{N_{R}}\right) \cosh (y \Gamma t)+\hat{\sigma} \sinh (y \Gamma t)+\hat{\mathcal{S}} \sin (x \Gamma t)+\hat{\mathcal{C}} \cos (x \Gamma t)},
\end{aligned}
$$

where

$$
\begin{aligned}
N_{R} & \equiv \frac{N_{(3) \perp, 4} \kappa_{(3) \perp, 4}}{N_{(1) \perp, 2} \kappa_{(1) \perp, 2}}, \\
\Delta X & \equiv \frac{1}{\sqrt{N_{R}}} \frac{X_{(1) \perp, 2}}{\kappa_{(1) \perp, 2}}-\sqrt{N_{R}} \frac{X_{(3) \perp, 4}}{\kappa_{(3) \perp, 4}} \quad(\text { for } X=\sigma, \mathcal{C}, \mathcal{S}), \\
\hat{X} & \equiv \frac{1}{\sqrt{N_{R}}} \frac{X_{(1) \perp, 2}}{\kappa_{(1) \perp, 2}}+\sqrt{N_{R}} \frac{X_{(3) \perp, 4}}{\kappa_{(3) \perp, 4}} \quad(\text { for } X=\sigma, \mathcal{C}, \mathcal{S}) .
\end{aligned}
$$

In eqs. (2.3)-(2.6), contribution from different overall factors in eq. (2.1) for two processes are taken into account. Taking the limit $N_{R} \rightarrow 1$ and $y \rightarrow 0$ in eq. (2.3), one finds an asymmetry whose overall normalization is eliminated, used in [4]. In eq. (2.5)-(2.6), $\Delta \mathcal{S}$ $(\Delta \mathcal{C})$ is equal to $\Delta S_{T}^{+}\left(\Delta C_{T}^{+}\right)$defined in [6] when one takes the limit $N_{R} \rightarrow 1$. In practice, we only need to consider the time difference $t$ within the interval $\left[0, \tau_{B}\right]$ where $\tau_{B}$ is the life time of $\mathrm{B}$ meson. Therefore, the approximation $\sinh (y \Gamma t) \simeq y \Gamma t, \cosh (y \Gamma t) \simeq 1$ is valid 
since $y \ll 1$ for neutral B meson system. Thus, we expand $A$ with respect to $y \Gamma t$,

$$
\begin{aligned}
A & \simeq \frac{\frac{1}{\sqrt{N_{R}}}-\sqrt{N_{R}}+\Delta \sigma y \Gamma t+\Delta \mathcal{S} \sin (x \Gamma t)+\Delta \mathcal{C} \cos (x \Gamma t)}{\frac{1}{\sqrt{N_{R}}}+\sqrt{N_{R}}+\hat{\sigma} y \Gamma t+\hat{\mathcal{S}} \sin (x \Gamma t)+\hat{\mathcal{C}} \cos (x \Gamma t)} \\
& =\frac{-\frac{\Delta N_{R}}{2}+\frac{\Delta \sigma}{2} y \Gamma t+\frac{\Delta \mathcal{S}}{2} \sin (x \Gamma t)+\frac{\Delta \mathcal{C}}{2} \cos (x \Gamma t)}{1+\frac{\hat{\sigma}}{2} y \Gamma t+\frac{\hat{\mathcal{S}}}{2} \sin (x \Gamma t)+\frac{\hat{\mathcal{C}}}{2} \cos (x \Gamma t)},
\end{aligned}
$$

where we denote

$$
N_{R}=1+\Delta N_{R}
$$

\section{Parameter definitions in terms of flavor based state}

In this section, we introduce parameters that reveal in the event number asymmetry in eq. (2.7) which we consider. In the processes which form the asymmetry, final states of B-decay are given as the same ones used for the BaBar experiment [4]. Mixing-parameters, $p, q, z, p_{K}, q_{K}$ and $z_{K}$ are defined in appendix B. Eqs. (B.3)-(B.4) lead the transformation property of mixing parameter as, $p \stackrel{\mathrm{CP} \text { or } \mathrm{T}}{\leftrightarrows} q, \quad p \stackrel{\mathrm{CPT}}{\longrightarrow} p, \quad q \stackrel{\mathrm{CPT}}{\longrightarrow} q$. Similarly, we obtain the transformation property of $z$ as, $z \stackrel{\mathrm{CP}}{\longrightarrow}-z, z \stackrel{\mathrm{T}}{\rightarrow}+z, z \stackrel{\mathrm{CPT}}{\longrightarrow}-z$. The transformation properties of $p_{K}, q_{K}$ and $z_{K}$ are the same as $p, q$ and $z$, respectively.

Following [6], we introduce B meson decay amplitudes and inverse decay amplitudes,

$$
A_{f} \equiv\left\langle f|T| B^{0}\right\rangle, \quad \bar{A}_{f} \equiv\left\langle f|T| \bar{B}^{0}\right\rangle, \quad A_{f}^{\mathrm{ID}} \equiv\left\langle B^{0}|T| f^{T}\right\rangle, \quad \bar{A}_{f}^{\mathrm{ID}} \equiv\left\langle\bar{B}^{0}|T| f^{T}\right\rangle,
$$

where $f^{T}$ is the time reversed state of $f$, i.e., the state with flipped momenta and spins. Note that $A_{f}\left(\bar{A}_{f}\right)$ and $A_{f}^{\mathrm{ID}}\left(\bar{A}_{f}^{\mathrm{ID}}\right)$ are exchanged under T-transformation. Throughout this paper, we introduce the notation $G_{f}, S_{f}$ and $C_{f}$ which are written in terms of amplitude ratio $\lambda_{f}$.

$$
\begin{aligned}
G_{f}=\frac{2 \operatorname{Re} \lambda_{f}}{1+\left|\lambda_{f}\right|^{2}}, \quad S_{f}=\frac{2 \operatorname{Im} \lambda_{f}}{1+\left|\lambda_{f}\right|^{2}}, \quad C_{f} & =\frac{1-\left|\lambda_{f}\right|^{2}}{1+\left|\lambda_{f}\right|^{2}}, \\
G_{f}^{2}+S_{f}^{2}+C_{f}^{2} & =1 .
\end{aligned}
$$

Using notation (3.1), we can denote following parameters,

$$
\begin{aligned}
\lambda_{\psi K_{S, L}} & \equiv \frac{q}{p} \frac{\bar{A}_{\psi K_{S, L}}}{A_{\psi K_{S, L}}} \sqrt{\frac{1+\theta_{\psi K_{S, L}}}{1-\theta_{\psi K_{S, L}}}}=\frac{q}{p} \frac{A_{\psi K_{S, L}}^{\mathrm{ID}}}{\bar{A}_{\psi K_{S, L}}^{\mathrm{ID}}} \sqrt{\frac{1-\theta_{\psi K_{S, L}}}{1+\theta_{\psi K_{S, L}}}} \\
\theta_{\psi K_{S, L}} & =\frac{A_{\psi K_{S, L}} A_{\psi K_{S, L}}^{\mathrm{ID}}-\bar{A}_{\psi K_{S, L}} \bar{A}_{\psi K_{S, L}}^{\mathrm{ID}}}{A_{\psi K_{S, L}} A_{\psi K_{S, L}}^{\mathrm{ID}}+\bar{A}_{\psi K_{S, L}} \bar{A}_{\psi K_{S, L}}^{\mathrm{ID}}} .
\end{aligned}
$$

Note that $\psi K_{L}$ and $\psi K_{S}$ are not exact CP eigenstates. $G_{\psi K_{S, L}}, S_{\psi K_{S, L}}$ and $C_{\psi K_{S, L}}$ are parameters written in terms of $\lambda_{\psi K_{S, L}} \cdot \theta_{\psi K_{S, L}}, G_{\psi K_{S, L}}, S_{\psi K_{S, L}}$ and $C_{\psi K_{S, L}}$ explicitly appear 
in coefficients of the master formula (A.1)-(A.5) for the processes. In eq. (3.4), $\lambda_{\psi K_{S, L}}$ is written in terms of the decay amplitude that the final state is the mass eigenstate $\psi K_{S, L}$. $A_{\psi K_{S, L}}^{(\mathrm{ID})}$ and $\bar{A}_{\psi K_{S, L}}^{(\mathrm{ID})}$ can be expanded with respect to amplitudes with a flavor eigenstate as $A_{\psi K^{0}}^{(\mathrm{ID})}, A_{\psi \bar{K}^{0}}^{(\mathrm{ID})}, \bar{A}_{\psi K^{0}}^{(\mathrm{ID})}$ and $\bar{A}_{\psi \bar{K}^{0}}^{(\mathrm{ID})}$. The expressions of $A_{\psi K_{S, L}}^{(\mathrm{ID})}$ and $\bar{A}_{\psi K_{S, L}}^{(\mathrm{ID})}$ are exhibited in eqs. (B.12)-(B.19). Note that the wrong strangeness decay amplitudes given as,

$$
A_{\psi \bar{K}^{0}}, \quad A_{\psi \bar{K}^{0}}^{\mathrm{ID}}, \quad \bar{A}_{\psi K^{0}}, \quad \bar{A}_{\psi K^{0}}^{\mathrm{ID}}
$$

are numerically smaller than the right strangeness decay amplitudes given as,

$$
A_{\psi K^{0}}, \quad A_{\psi K^{0}}^{\mathrm{ID}}, \quad \bar{A}_{\psi \bar{K}^{0}}, \quad \bar{A}_{\psi \bar{K}^{0}}^{\mathrm{ID}}
$$

for the standard model. Thus, we ignore terms with higher power of $A_{\psi \bar{K}^{0}}^{(\mathrm{ID})}$ and $\bar{A}_{\psi K^{0}}^{\text {(ID) }}$. Using eqs. (B.12)-(B.19), we can calculate $\theta_{\psi K_{S, L}}$ as,

$$
\begin{aligned}
\theta_{\psi K_{S}} & \simeq \theta_{K}-z_{K}, \quad \theta_{\psi K_{L}} \simeq \theta_{K}+z_{K} \\
\theta_{K} & =\frac{A_{\psi K^{0}} A_{\psi K^{0}}-\bar{A}_{\psi \bar{K}^{0}} \bar{A}_{\psi \bar{K}^{0}}^{\mathrm{ID}}}{A_{\psi K^{0}} A_{\psi K^{0}}^{\mathrm{ID}}+\bar{A}_{\psi \bar{K}^{0}} \bar{A}_{\psi \bar{K}^{0}}^{\mathrm{ID}}},
\end{aligned}
$$

where $\theta_{K}$ expresses $\mathrm{CP}$ and $\mathrm{CPT}$ violation in right strangeness decays of $\mathrm{B}$ meson and it corresponds to $\hat{\theta}_{\psi K}$ of [6]. Note that in [6] indirect CPT violation $z_{K}$ is not taken into account. When deriving eq. (3.8), we calculated at linear order approximation with respect to $z_{K}, \theta_{K}$ and wrong strangeness decay amplitudes. Then, we can write $\lambda_{\psi K_{S, L}}$ as,

$$
\begin{aligned}
\lambda_{\psi K_{S}} & \simeq \lambda\left(1-\Delta \lambda_{\mathrm{wst}}\right), \quad \lambda_{\psi K_{L}} \simeq-\lambda\left(1+\Delta \lambda_{\mathrm{wst}}\right), \\
\lambda & \equiv \frac{q}{p} \frac{p_{K}}{q_{K}} \frac{\bar{A}_{\psi \bar{K}^{0}}}{A_{\psi K^{0}}} \sqrt{\frac{1+\theta_{K}}{1-\theta_{K}}}=\frac{q}{p} \frac{p_{K}}{q_{K}} \frac{A_{\psi K^{0}}^{\mathrm{ID}}}{\bar{A}_{\psi \bar{K}^{0}}^{\mathrm{ID}}} \sqrt{\frac{1-\theta_{K}}{1+\theta_{K}}},
\end{aligned}
$$

where $\Delta \lambda_{\text {wst }}$ consists of wrong strangeness decays as,

$$
\begin{aligned}
\Delta \lambda_{\mathrm{wst}} & =\lambda_{\psi \bar{K}^{0}}^{\mathrm{wst}}-\bar{\lambda}_{\psi K^{0}}^{\mathrm{wst}}, \\
\lambda_{\psi \bar{K}^{0}}^{\mathrm{wst}} & \equiv \frac{p_{K}}{q_{K}} \frac{A_{\psi \bar{K}^{0}}}{A_{\psi K^{0}}} \sqrt{\frac{1+\theta_{\psi K^{0}}}{1-\theta_{\psi K^{0}}}}=\frac{p_{K}}{q_{K}} \frac{\bar{A}_{\psi K^{0}}^{\mathrm{ID}}}{\bar{A}_{\psi \bar{K}^{0}}^{\mathrm{ID}}} \sqrt{\frac{1-\theta_{\psi K^{0}}}{1+\theta_{\psi K^{0}}}}, \\
\bar{\lambda}_{\psi K^{0}}^{\mathrm{wst}} & \equiv \frac{q_{K}}{p_{K}} \frac{\bar{A}_{\psi K^{0}}}{\bar{A}_{\psi \bar{K}^{0}}} \sqrt{\frac{1+\bar{\theta}_{\psi \bar{K}^{0}}}{1-\bar{\theta}_{\psi \bar{K}^{0}}}}=\frac{q_{K}}{p_{K}} \frac{A_{\psi \bar{K}^{0}}^{\mathrm{ID}}}{A_{\psi K^{0}}^{\mathrm{ID}}} \sqrt{\frac{1-\bar{\theta}_{\psi \bar{K}^{0}}}{1+\bar{\theta}_{\psi \bar{K}^{0}}}}, \\
\theta_{\psi K^{0}} & \equiv \frac{A_{\psi K^{0}} \bar{A}_{\psi K^{0}}^{\mathrm{ID}}-A_{\psi \bar{K}^{0}} \bar{A}_{\psi \bar{K}^{0}}^{\mathrm{ID}}}{A_{\psi K^{0}} \bar{A}_{\psi K^{0}}^{\mathrm{ID}}+A_{\psi \bar{K}^{0}} \bar{A}_{\psi \bar{K}^{0}}^{\mathrm{ID}}}, \quad \bar{\theta}_{\psi \bar{K}^{0}} \equiv \frac{\bar{A}_{\psi \bar{K}^{0}} A_{\psi \bar{K}^{0}}-\bar{A}_{\psi K^{0}} A_{\psi K^{0}}^{\mathrm{ID}}}{\bar{A}_{\psi \bar{K}^{0}} A_{\psi \bar{K}^{0}}^{\mathrm{ID}}+\bar{A}_{\psi K^{0}} A_{\psi K^{0}}^{\mathrm{ID}}}
\end{aligned}
$$

where eq. (3.15) describes CPT violation in wrong strangeness decays. Similar to eq. (3.12), we can define a parameter including wrong sign decay amplitudes as,

$$
\hat{\lambda}_{\mathrm{wst}}=\lambda_{\psi \bar{K}^{0}}^{\mathrm{wst}}+\bar{\lambda}_{\psi K^{0}}^{\mathrm{wst}}
$$


Since wrong sign semi-leptonic decay amplitudes and CPT violation, $\theta_{\psi K^{0}}$ and $\bar{\theta}_{\psi \bar{K}^{0}}$, are small, we can expand eqs. (3.13), (3.14) as,

$$
\lambda_{\psi K^{0}}^{\mathrm{wst}} \simeq \frac{p_{K}}{q_{K}} \frac{A_{\psi \bar{K}^{0}}}{A_{\psi K^{0}}} \simeq \frac{p_{K}}{q_{K}} \frac{\bar{A}_{\psi K^{0}}^{\mathrm{ID}}}{\bar{A}_{\psi \bar{K}^{0}}^{\mathrm{ID}}}, \quad \bar{\lambda}_{\psi K^{0}}^{\mathrm{wst}} \simeq \frac{q_{K}}{p_{K}} \frac{\bar{A}_{\psi K^{0}}}{\bar{A}_{\psi \bar{K}^{0}}} \simeq \frac{q_{K}}{p_{K}} \frac{A_{\psi \bar{K}^{0}}^{\mathrm{ID}}}{A_{\psi K^{0}}^{\mathrm{ID}}} .
$$

Eq. (3.10) indicates that $\lambda_{\psi K_{S, L}}$ is composed of the leading part $\lambda$ and the sub-leading part suppressed by wrong strangeness decay amplitude. Taking the CPT conserving limit in eq. (3.10), one can obtain the relation in ref. [7]. Note that $\lambda$ has the definitive transformation property of $\mathrm{T}, \mathrm{CP}$ and $\mathrm{CPT}$, such as $\lambda \stackrel{\mathrm{T}}{\rightarrow}(\lambda)^{-1}, \lambda \stackrel{\mathrm{CP}}{\longrightarrow}(\lambda)^{-1}, \lambda \stackrel{\mathrm{CPT}}{\longrightarrow} \lambda$. One introduces $G, S$ and $C$ analogous to eq. (3.2) by replacing $\lambda_{f}$ with $\lambda$. They are transformed under $\mathrm{T}$ as,

$$
\begin{aligned}
& G=\frac{2 \operatorname{Re} \lambda}{1+|\lambda|^{2}} \stackrel{\mathrm{T}}{\rightarrow} \frac{2 \operatorname{Re}(1 / \lambda)}{1+|1 / \lambda|^{2}}=\frac{2 \operatorname{Re} \lambda^{*}}{|\lambda|^{2}+1}=+G, \\
& S=\frac{2 \operatorname{Im} \lambda}{1+|\lambda|^{2}} \stackrel{\mathrm{T}}{\rightarrow} \frac{2 \operatorname{Im}(1 / \lambda)}{1+|1 / \lambda|^{2}}=\frac{2 \operatorname{Im} \lambda^{*}}{|\lambda|^{2}+1}=-S, \\
& C=\frac{1-|\lambda|^{2}}{1+|\lambda|^{2}} \stackrel{\mathrm{T}}{\rightarrow} \frac{1-|1 / \lambda|^{2}}{1+|1 / \lambda|^{2}}=\frac{|\lambda|^{2}-1}{|\lambda|^{2}+1}=-C .
\end{aligned}
$$

The CP transformation property of $G, S, C$ is the same as (3.18)-(3.20). Thus, the CPT transformation property is also determined as $G \stackrel{\mathrm{CPT}}{\longrightarrow}+G, S \stackrel{\mathrm{CPT}}{\longrightarrow}+S, C \stackrel{\mathrm{CPT}}{\longrightarrow}+C \cdot|\lambda|$ is close to 1 since deviation of $|q / p|,\left|p_{K} / q_{K}\right|$ and $\left|\bar{A}_{\psi \bar{K}^{0}} / A_{\psi K^{0}}\right|$ from 1 is small. Hence, we can find that $C$ is a small parameter. One can also derive the transformation property of eqs. (3.13)-(3.14) such like $\lambda_{\psi K^{0}}^{\text {wst }} \stackrel{\mathrm{T}}{\rightarrow} \bar{\lambda}_{\psi K^{0}}^{\text {wst }}, \lambda_{\psi K^{0}}^{\text {wst }} \stackrel{\mathrm{CP}}{\longrightarrow} \bar{\lambda}_{\psi K^{0}}^{\text {wst }}$ and $\lambda_{\psi K^{0}}^{\text {wst }} \stackrel{\mathrm{CPT}}{\longrightarrow} \lambda_{\psi K^{0}}^{\text {wst }}$. Therefore, the parameters in eqs. (3.12), (3.16) are transformed as,

$$
\Delta \lambda_{\text {wst }} \stackrel{\mathrm{T}}{\rightarrow}-\Delta \lambda_{\text {wst }}, \quad \hat{\lambda}_{\text {wst }} \stackrel{\mathrm{T}}{\rightarrow} \hat{\lambda}_{\text {wst }} .
$$

The CP transformation property of the parameter (3.12), (3.16) is the same as eq. (3.21).

Note that parameters $G_{\psi K_{S, L}}, S_{\psi K_{S, L}}$ and $C_{\psi K_{S, L}}$ are related with the parameters $G, S$ and $C$ as,

$$
\begin{array}{rlrl}
G_{\psi K_{S}} & \simeq G+S \Delta \lambda_{\mathrm{wst}}^{I}, & & G_{\psi K_{L}} \simeq-\left(G-S \Delta \lambda_{\mathrm{wst}}^{I}\right), \\
S_{\psi K_{S}} \simeq S-G \Delta \lambda_{\mathrm{wst}}^{I}, & & S_{\psi K_{L}} \simeq-\left(S+G \Delta \lambda_{\mathrm{wst}}^{I}\right), \\
C_{\psi K_{S}} \simeq C+\Delta \lambda_{\mathrm{wst}}^{R}, & C_{\psi K_{L}} \simeq C-\Delta \lambda_{\mathrm{wst}}^{R},
\end{array}
$$

where we use notation for an arbitrary complex number $A, A^{R} \equiv \operatorname{Re} A, A^{I} \equiv \operatorname{Im} A$, throughout this paper. When deriving eqs. (3.22)-(3.24), we ignored higher order terms of $C$ and

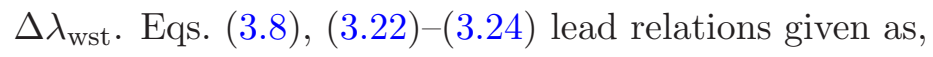

$$
\begin{aligned}
& \theta_{\psi K_{S}}+\theta_{\psi K_{L}}=2 \theta_{K}, \quad \theta_{\psi K_{S}}-\theta_{\psi K_{L}}=-2 z_{K}, \\
& G_{\psi K_{S}}-G_{\psi K_{L}}=2 G, \quad S_{\psi K_{S}}-S_{\psi K_{L}}=2 S, \quad C_{\psi K_{S}}+C_{\psi K_{L}}=2 C, \\
& G_{\psi K_{S}}+G_{\psi K_{L}}=2 S \Delta \lambda_{\mathrm{wst}}^{I}, \quad S_{\psi K_{S}}+S_{\psi K_{L}}=-2 G \Delta \lambda_{\mathrm{wst}}^{I}, \quad C_{\psi K_{S}}-C_{\psi K_{L}}=2 \Delta \lambda_{\mathrm{wst}}^{R} .
\end{aligned}
$$


Since we have included the effect of indirect $\mathrm{CP}$ violation of Kaon system, we show how the correction due to $\epsilon_{K}$ arises. While the expression of $G, C$ and $S$ in eqs. (3.18)-(3.20) is invariant under the arbitrary large rephasing such as $\left\langle K^{0}\right| \rightarrow e^{-i \alpha_{K}}\left\langle K^{0}\right|$ and $\left\langle\bar{K}^{0}\right| \rightarrow$ $e^{i \alpha_{K}}\left\langle\bar{K}^{0}\right|$, the parametrization with $\epsilon_{K} \ll 1$ allows only the small rephasing $\alpha_{K} \ll 1$.

$$
\frac{p_{K}}{q_{K}}=\frac{1+\epsilon_{K}}{1-\epsilon_{K}} \simeq 1+2 \epsilon_{K} .
$$

Keeping only the terms which are linear to $\epsilon_{K}, G, S$ and $C$ are expanded as follows,

$$
\begin{aligned}
G & =G^{\prime}-2 S^{\prime} \epsilon_{K}^{I}, \\
S & =S^{\prime}+2 G^{\prime} \epsilon_{K}^{I}, \\
C & =C^{\prime}-2 \epsilon_{K}^{R},
\end{aligned}
$$

where $G^{\prime}, S^{\prime}$ and $C^{\prime}$ are obtained by taking the limit $\frac{p_{K}}{q_{K}} \rightarrow 1$ in $G, S$ and $C$. Namely, they are defined by replacing $\lambda$ with $\lambda^{\prime}$ in the expression for $G, S$ and $C$.

$$
\lambda^{\prime}=\frac{q}{p} \frac{\bar{A}_{\psi \bar{K}^{0}}}{A_{\psi K^{0}}} \sqrt{\frac{1+\theta_{K}}{1-\theta_{K}}}, \quad C^{\prime}=\frac{1-\left|\lambda^{\prime}\right|^{2}}{1+\left|\lambda^{\prime}\right|^{2}} .
$$

As shown in table (1), $\left(G^{\prime}, S^{\prime}, C^{\prime}\right)$ equal to $\left(\hat{G}_{\psi K}, \hat{S}_{\psi K}, \hat{C}_{\psi K}\right)$ defined in [6] where indirect $\mathrm{CP}$ violation $\epsilon_{K}$ is neglected. When one changes the phase convention of states as, the phase of $\lambda^{\prime}$ changes as follows,

$$
\lambda^{\prime} \rightarrow \lambda^{\prime} e^{2 i \alpha_{K}} .
$$

Assuming the phase $\alpha_{K}$ is small, $G^{\prime}, S^{\prime}$, and $\epsilon_{K}^{I}$ change as,

$$
\begin{aligned}
G^{\prime} & \rightarrow G^{\prime}-2 \alpha_{K} S^{\prime}, \\
S^{\prime} & \rightarrow S^{\prime}+2 \alpha_{K} G^{\prime}, \\
\epsilon_{K}^{I} & \rightarrow \epsilon_{K}^{I}-\alpha_{K},
\end{aligned}
$$

while $C^{\prime}$ and $\epsilon_{K}^{R}$ are invariant,

$$
C^{\prime} \rightarrow C^{\prime}, \quad \epsilon_{K}^{R} \rightarrow \epsilon_{K}^{R}
$$

Hereafter, we expand $C$ in terms of $C^{\prime}$ and $\epsilon_{K}^{R}$ as shown in eq. (3.29) and we do not expand $S$ and $G$ since they are invariant under the rephasing. The numerical significance of $\epsilon_{K}^{R}$ will be discussed in the next section.

We turn to definition for parameters including semi-leptonic decay amplitudes of B meson. In the following, from eq. (3.34) to eq. (3.38), we adopt the notations of [6]. Right sign semi-leptonic decay amplitudes are denoted as,

$$
\begin{array}{ll}
A_{l^{+}}=\left\langle l^{+} X|T| B^{0}\right\rangle, & A_{l^{+}}^{\mathrm{ID}}=\left\langle B^{0}|T|\left(l^{+} X\right)^{T}\right\rangle, \\
\bar{A}_{l^{-}}=\left\langle l^{-} X|T| \bar{B}^{0}\right\rangle, & \bar{A}_{l^{-}}^{\mathrm{ID}}=\left\langle\bar{B}^{0}|T|\left(l^{-} X\right)^{T}\right\rangle .
\end{array}
$$

Wrong sign semi-leptonic decay amplitudes are similarly given as,

$$
\begin{aligned}
A_{l^{-}} & =\left\langle l^{-} X|T| B^{0}\right\rangle, & A_{l^{-}}^{\mathrm{ID}} & =\left\langle B^{0}|T|\left(l^{-} X\right)^{T}\right\rangle, \\
\bar{A}_{l^{+}} & =\left\langle l^{+} X|T| \bar{B}^{0}\right\rangle, & \bar{A}_{l^{+}}^{\mathrm{ID}} & =\left\langle\bar{B}^{0}|T|\left(l^{+} X\right)^{T}\right\rangle .
\end{aligned}
$$




\begin{tabular}{|c|c|c|c|}
\hline Notation of this paper & Notation of [6] & Notatin of [6] & Notation of this paper \\
\hline$\lambda_{\psi K_{S}}$ & $\frac{p_{K}}{q_{K}} \lambda_{\psi K_{1}}$ & $\lambda_{\psi K_{1}}$ & $\lambda^{\prime}\left(1-\Delta \lambda_{w s t}\right)$ \\
\hline$\lambda_{\psi K_{L}}$ & $\frac{p_{K}}{q_{K}} \lambda_{\psi K_{2}}$ & $\lambda_{\psi K_{2}}$ & $-\lambda^{\prime}\left(1+\Delta \lambda_{w s t}\right)$ \\
\hline$G_{\psi K_{S}}$ & $G_{\psi K_{1}}-2 S_{\psi K_{1}} \epsilon_{K}^{I}$ & $\hat{G}_{\psi K}=\frac{G_{\psi K_{1}}-G_{\psi K_{2}}}{2}$ & $G^{\prime}$ \\
\hline$S_{\psi K_{S}}$ & $S_{\psi K_{1}}+2 G_{\psi K_{1}} \epsilon_{K}^{I}$ & $\hat{S}_{\psi K}=\frac{S_{\psi K_{1}}-S_{\psi K_{2}}}{2}$ & $S^{\prime}$ \\
\hline$C_{\psi K_{S}}$ & $C_{\psi K_{1}}-2 \epsilon_{K}^{R}$ & $\hat{C}_{\psi K}=\frac{C_{\psi K_{1}}+C_{\psi K_{2}}}{2}$ & $C^{\prime}$ \\
\hline$G_{\psi K_{L}}$ & $G_{\psi K_{2}}-2 S_{\psi K_{2}} \epsilon_{K}^{I}$ & $\Delta G_{\psi K}=\frac{G_{\psi K_{1}}+G_{\psi K_{2}}}{2}$ & $S^{\prime} \Delta \lambda_{w s t}^{I}$ \\
\hline$S_{\psi K_{L}}$ & $S_{\psi K_{2}}+2 G_{\psi K_{2}} \epsilon_{K}^{I}$ & $\Delta S_{\psi K}=\frac{S_{\psi K_{1}}+S_{\psi K_{2}}}{2}$ & $-G^{\prime} \Delta \lambda_{w s t}^{I}$ \\
\hline$C_{\psi K_{L}}$ & $C_{\psi K_{2}}-2 \epsilon_{K}^{R}$ & $\Delta C_{\psi K}=\frac{C_{\psi K_{1}}-C_{\psi K_{2}}}{2}$ & $\Delta \lambda_{w s t}^{R}$ \\
\hline$\theta_{K}$ & $\hat{\theta}_{\psi K}=\frac{\theta_{\psi K 1}+\theta_{\psi K 2}}{2}$ & $\Delta \theta_{\psi K}=\frac{\theta_{\psi K 1}-\theta_{\psi K 2}}{2}$ & 0 \\
\hline
\end{tabular}

Table 1. The correspondence of parameters in this paper and those of [6]. In this paper, $\psi K_{1}$ corresponds to $\psi K_{S}$ and $\psi K_{2}$ corresponds to $\psi K_{L}$ respectively in [6] where indirect CP violation parameter $\epsilon_{K}$ is neglected. In this paper, $\psi K_{L}$ and $\psi K_{S}$ include the effect of indirect CP and CPT violation. The first column shows the quantities defined for mass eigenstates $\left(K_{L}, K_{S}\right)$. From the third row to the eighth row in the second column, the quantities in the first column are expanded up to the first order of $\epsilon_{K}$ and are written in terms of the quantities for CP eigenstates $K_{1}, K_{2}$. In the third column and in the fourth column, we show how $\left(\hat{G}_{\psi K}, \hat{S}_{\psi K}, \hat{C}_{\psi K}\right)$ and $\left(\Delta G_{\psi K}, \Delta S_{\psi K}, \Delta C_{\psi K}\right)$ in [6] are related to $\left(G^{\prime}, S^{\prime}, C^{\prime}, \Delta \lambda_{w s t}\right)$ defined in this paper. About CPT violation parameter of strangeness changing decay, one can show $\theta_{\psi K 1}=\theta_{\psi K 2}=\theta_{K}$. Therefore $\hat{\theta}_{\psi K}=\theta_{K}$ and $\Delta \theta_{\psi K}=0$.

For the case of the standard model, wrong sign semi-leptonic decay amplitudes are smaller than right sign decay amplitudes. Thus, we ignore higher powers of wrong sign decay amplitudes than linear order. We define parameters including semi-leptonic decay amplitudes as,

$$
\begin{aligned}
& \lambda_{l^{+}} \equiv \frac{q}{p} \frac{\bar{A}_{l^{+}}}{A_{l^{+}}} \sqrt{\frac{1+\theta_{l^{+}}}{1-\theta_{l^{+}}}}=\frac{q}{p} \frac{A_{l^{-}}^{\mathrm{ID}}}{\bar{A}_{l^{-}}^{\mathrm{ID}}} \sqrt{\frac{1-\theta_{l^{+}}}{1+\theta_{l^{+}}}}, \quad \theta_{l^{+}}=\frac{A_{l^{+}} A_{l^{-}}^{\mathrm{ID}}-\bar{A}_{l^{+}} \bar{A}_{l^{-}}^{\mathrm{ID}}}{A_{l^{+}} A_{l^{-}}^{\mathrm{ID}}+\bar{A}_{l^{+}} \bar{A}_{l^{-}}^{\mathrm{ID}}} \\
& \lambda_{l^{-}} \equiv \frac{q}{p} \frac{\bar{A}_{l^{-}}}{A_{l^{-}}} \sqrt{\frac{1+\theta_{l^{-}}}{1-\theta_{l^{-}}}}=\frac{q}{p} \frac{A_{l^{+}}^{\mathrm{ID}}}{\bar{A}_{l^{+}}^{\mathrm{ID}}} \sqrt{\frac{1-\theta_{l^{-}}}{1+\theta_{l^{-}}}}, \quad \theta_{l^{-}}=\frac{A_{l^{-}} A_{l^{+}}^{\mathrm{ID}}-\bar{A}_{l^{-}} \bar{A}_{l^{+}}^{\mathrm{ID}}}{A_{l^{-}} A_{l^{+}}^{\mathrm{ID}}-\bar{A}_{l^{-}} \bar{A}_{l^{+}}^{\mathrm{ID}}}
\end{aligned}
$$

where $\theta_{l^{ \pm}}$expresses CPT violation in semi-leptonic decays of B meson. One can find the transformation law such like $\lambda_{l^{+}} \stackrel{T}{\rightarrow}\left(\lambda_{l^{-}}\right)^{-1}, \lambda_{l^{+}} \stackrel{C P}{\longrightarrow}\left(\lambda_{l^{-}}\right)^{-1}, \lambda_{l^{+}} \stackrel{C P T}{\longrightarrow} \lambda_{l^{+}}$by its definition (3.36)-(3.37). We assume that CPT violating parameter $\theta_{l^{ \pm}}$is small. At linear order approximation of $\theta_{l^{ \pm}}$and wrong sign semi-leptonic decay amplitudes, we obtain,

$$
\lambda_{l^{+}} \simeq \frac{q}{p} \frac{\bar{A}_{l^{+}}}{A_{l^{+}}} \simeq \frac{q}{p} \frac{A_{l^{-}}^{\mathrm{ID}}}{\bar{A}_{l^{-}}^{\mathrm{ID}}}, \quad \lambda_{l^{-}}^{-1} \simeq \frac{p}{q} \frac{A_{l^{-}}}{\bar{A}_{l^{-}}} \simeq \frac{p}{q} \frac{\bar{A}_{l^{+}}^{\mathrm{ID}}}{A_{l^{+}}^{\mathrm{ID}}},
$$

where we can see that contribution of $\theta_{l^{ \pm}}$approximately vanishes in eq. (3.38). Following ref. [6], we also define $G_{l^{ \pm}}, S_{l^{ \pm}}$and $C_{l^{ \pm}}$analogous to eq. (3.2) by replacing $\lambda_{f}$ with $\lambda_{l^{ \pm}}$. The parameters $G_{l^{ \pm}}, S_{l^{ \pm}}$and $C_{l^{ \pm}}$explicitly appear in coefficients of master formula (A.1)-(A.5) 
for the processes in which the final states are given as $l^{ \pm} X$. Eq. (3.38) gives approximate expressions for $G_{l^{ \pm}}, S_{l^{ \pm}}$and $C_{l^{ \pm}}$as,

$$
\begin{aligned}
G_{l^{+}} & =\frac{2 \operatorname{Re} \lambda_{l^{+}}}{1+\left|\lambda_{l^{+}}\right|^{2}} \simeq 2 \operatorname{Re} \lambda_{l^{+}}, \quad G_{l^{-}}=\frac{2 \operatorname{Re} \lambda_{l^{-}}}{1+\left|\lambda_{l^{-}}\right|^{2}} \simeq 2 \operatorname{Re}\left(\lambda_{l^{-}}^{-1}\right), \quad C_{l^{ \pm}}=\frac{1-\left|\lambda_{l^{ \pm}}\right|^{2}}{1+\left|\lambda_{l^{ \pm}}\right|^{2}} \simeq \pm 1, \\
S_{l^{+}} & =\frac{2 \operatorname{Im} \lambda_{l^{+}}}{1+\left|\lambda_{l^{+}}\right|^{2}} \simeq 2 \operatorname{Im} \lambda_{l^{+}}, \quad S_{l^{-}}=\frac{2 \operatorname{Im} \lambda_{l^{-}}}{1+\left|\lambda_{l^{-}}\right|^{2}} \simeq-2 \operatorname{Im}\left(\lambda_{l^{-}}^{-1}\right) .
\end{aligned}
$$

Note that eq. (3.39) implies that $G_{l^{ \pm}}$and $S_{l^{ \pm}}$are small numbers since $\lambda_{l^{+}}$and $\lambda_{l^{-}}^{-1}$ are suppressed. We can find the relations,

$$
\begin{array}{ll}
G_{l^{+}}+G_{l^{-}}=2 \hat{\lambda}_{l}^{R}, & S_{l^{+}}-S_{l^{-}}=2 \hat{\lambda}_{l}^{I}, \\
G_{l^{+}}-G_{l^{-}}=2 \Delta \lambda_{l}^{R}, & S_{l^{+}}+S_{l^{-}}=2 \Delta \lambda_{l}^{I},
\end{array}
$$

where $\hat{\lambda}_{l}$ and $\Delta \lambda_{l}$ are defined as,

$$
\hat{\lambda}_{l} \equiv \lambda_{l^{+}}+\lambda_{l^{-}}^{-1}, \quad \Delta \lambda_{l} \equiv \lambda_{l^{+}}-\lambda_{l^{-}}^{-1} .
$$

They transform definitively under CP, T and CPT. One obtains the transformation property of $\mathrm{T}$ as,

$$
\hat{\lambda}_{l} \stackrel{\mathrm{T}}{\rightarrow}\left(\lambda_{l^{-}}\right)^{-1}+\lambda_{l^{+}}=+\hat{\lambda}_{l}, \quad \Delta \lambda_{l} \stackrel{\mathrm{T}}{\rightarrow}\left(\lambda_{l^{-}}\right)^{-1}-\lambda_{l^{+}}=-\Delta \lambda_{l} .
$$

The CP transformation property of $\hat{\lambda}_{l}$ and $\Delta \lambda_{l}$ is the same as (3.43). Hence, the CPT transformation property of $\hat{\lambda}_{l}$ and $\Delta \lambda_{l}$ is also determined as $\hat{\lambda}_{l} \stackrel{\mathrm{CPT}}{\longrightarrow} \hat{\lambda}_{l}, \Delta \lambda_{l} \stackrel{\mathrm{CPT}}{\longrightarrow} \Delta \lambda_{l}$. Eqs. (3.22)-(3.24), (3.40)-(3.41) enable one to write down the asymmetry in eq. (2.3) for the BaBar experiment in terms of parameters which are exactly T-odd or T-even. Similarly, one defines,

$$
\begin{aligned}
R_{M} & \equiv \frac{|p|^{2}-|q|^{2}}{|p|^{2}+|q|^{2}}, \quad \xi_{l} \equiv \frac{\bar{A}_{l^{-}} A_{l^{+}}^{\mathrm{ID}}-A_{l^{+}} \bar{A}_{l^{-}}^{\mathrm{ID}}}{\bar{A}_{l^{-}} A_{l^{+}}^{\mathrm{ID}}+A_{l^{+}} \bar{A}_{l^{-}}^{\mathrm{ID}}}, \quad C_{\xi}^{l}, \equiv \frac{1-\left|\lambda_{\xi}^{l}\right|^{2}}{1+\left|\lambda_{\xi}^{l}\right|^{2}}, \\
\lambda_{\xi}^{l} & \equiv \frac{A_{l^{+}}}{\bar{A}_{l^{-}}} \sqrt{\frac{1+\xi_{l}}{1-\xi_{l}}}=\frac{A_{l^{+}}^{\mathrm{ID}}}{\bar{A}_{l^{-}}^{\mathrm{ID}}} \sqrt{\frac{1-\xi_{l}}{1+\xi_{l}}} .
\end{aligned}
$$

In eq. (3.44), $R_{M}$ expresses mixing-induced $\mathrm{CP}$ and $\mathrm{T}$ violation for $\mathrm{B}$ meson system [6] and is a small number. As for newly introduced parameters, $\xi_{l}$ implies $\mathrm{CP}$ and $\mathrm{T}$ violation in right sign semi-leptonic decays and we also assume $\xi_{l}$ is a small number. The expression of $\lambda_{\xi}^{l}$ (3.45) includes right sign semi-leptonic decay amplitude ratio and we assume $\left|A_{l^{+}} / \bar{A}_{l^{-}}\right| \simeq 1$. Therefore, $C_{\xi}^{l}$ is a small number compared with $\mathcal{O}(1)$. The parameters defined in eq. (3.44) also appear in the asymmetry in eq. (2.3).

Hereafter, we describe some significant points of the parameters defined in this section. Note that the parameters given as,

$$
S, C, G, \theta_{K}, R_{M}, z, z_{K}, \hat{\lambda}_{l}, \Delta \lambda_{l}, \xi_{l}, C_{\xi}^{l}, \hat{\lambda}_{\mathrm{wst}} \text { and } \Delta \lambda_{\mathrm{wst}},
$$

have definitive transformation properties exhibited in table 2. In the processes which we consider, $K_{S, L}$ is included as a final state, and the effect of mixing induced $\mathrm{T}$ and $\mathrm{CP}$ 


\begin{tabular}{|c|c|c|c|c|c|c|c|c|c|c|c|c|c|}
\hline & $S$ & $C$ & $G$ & $\theta_{K}$ & $R_{M}$ & $z$ & $z_{K}$ & $\hat{\lambda}_{l}$ & $\Delta \lambda_{l}$ & $\xi_{l}$ & $C_{\xi}^{l}$ & $\hat{\lambda}_{\text {wst }}$ & $\Delta \lambda_{\text {wst }}$ \\
\hline $\mathrm{T}$ & - & - & + & + & - & + & + & + & - & - & + & + & - \\
\hline $\mathrm{CP}$ & - & - & + & - & - & - & - & + & - & - & - & + & - \\
\hline $\mathrm{CPT}$ & + & + & + & - & + & - & - & + & + & + & - & + & + \\
\hline
\end{tabular}

Table 2. Transformation property of the parameters definitively transformed under T, CP and CPT.

\begin{tabular}{|c|c|c|c|c|c|c|c|c|}
\hline & $p / q$ & $p_{K} / q_{K}$ & $\theta_{\psi K^{0}}$ & $\theta_{l^{+}}$ & $\lambda$ & $\lambda_{\psi K^{0}}^{\mathrm{wst}}$ & $\lambda_{l^{+}}$ & $\lambda_{\xi}^{l}$ \\
\hline $\mathrm{T}$ & $q / p$ & $q_{K} / p_{K}$ & $-\bar{\theta}_{\psi \bar{K}^{0}}$ & $\theta_{l^{-}}$ & $(\lambda)^{-1}$ & $\bar{\lambda}_{\psi K^{0}}^{\mathrm{wst}}$ & $\left(\lambda_{l^{-}}\right)^{-1}$ & $\lambda_{\xi}^{l}$ \\
\hline $\mathrm{CP}$ & $q / p$ & $q_{K} / p_{K}$ & $\bar{\theta}_{\psi \bar{K}^{0}}$ & $-\theta_{l^{-}}$ & $(\lambda)^{-1}$ & $\bar{\lambda}_{\psi K^{0}}^{\mathrm{wst}}$ & $\left(\lambda_{l^{-}}\right)^{-1}$ & $\left(\lambda_{\xi}^{l}\right)^{-1}$ \\
\hline $\mathrm{CPT}$ & $p / q$ & $p_{K} / q_{K}$ & $-\theta_{\psi K^{0}}$ & $-\theta_{l^{+}}$ & $\lambda$ & $\lambda_{\psi \bar{K}^{0}}^{\mathrm{wst}}$ & $\lambda_{l^{+}}$ & $\left(\lambda_{\xi}^{l}\right)^{-1}$ \\
\hline
\end{tabular}

Table 3. Transformation property of the parameters devoted to keep the definitive transformation property of the parameters in table 2 .

violation, $p_{K} / q_{K}$, appears in the expressions of $G, S, C, \hat{\lambda}_{\text {wst }}$ and $\Delta \lambda_{\text {wst }}$. Mixing-induced $\mathrm{CP}$ and $\mathrm{CPT}$ violation in $\mathrm{K}$ meson system, $z_{K}$, reveals in the asymmetry as well. In the next section, the asymmetry is written in terms of parameters (3.46) and it can be explicitly separated as T-odd parts and T-even parts.

The parameters defined as,

$$
p / q, p_{K} / q_{K}, \theta_{\psi K^{0}}, \bar{\theta}_{\psi \bar{K}^{0}}, \theta_{l^{ \pm}}, \lambda, \lambda_{\psi \bar{K}^{0}}^{\mathrm{wst}}, \bar{\lambda}_{\psi K^{0}}^{\mathrm{wst}}, \lambda_{l^{ \pm}} \text {, and } \lambda_{\xi}^{l},
$$

are dedicated to keep the definitive transformation property of parameters that reveal in table 2. The transformation property of the parameters (3.47) is exhibited in table 3 .

The parameters given as,

$$
\theta_{\psi K^{0}}, \bar{\theta}_{\psi \bar{K}^{0}}, \theta_{l^{ \pm}}, C, \theta_{K}, R_{M}, z, z_{K}, \hat{\lambda}_{l}, \Delta \lambda_{l}, \xi_{l}, C_{\xi}^{l}, \hat{\lambda}_{\mathrm{wst}} \text { and } \Delta \lambda_{\mathrm{wst}},
$$

are small numbers, and our calculation is based on linear order approximation with respect to the parameters (3.48) throughout this paper.

\section{Time dependent asymmetry including the overall constants}

In this section, we apply the event number asymmetry defined in eq. (2.7) to processes for B-meson decays. One should be aware of that the asymmetry considered in this paper includes the effect of different normalization for two rates; non-zero value of $\Delta N_{R}$ defined in eq. (2.8). As the BaBar asymmetry investigated in [4], we also assign $f_{1}, f_{2}, f_{3}, f_{4}$ with $\psi K_{L}, l^{-} X, l^{+} X, \psi K_{S}$, respectively. We call this process as I. We also consider the other three processes which can be obtained by interchanging $l^{-} X$ with $l^{+} X$ and $\psi K_{S}$ with $\psi K_{L}$ 
in the process I. Therefore we identify the four processes as,

$$
\begin{aligned}
\text { (I) } \quad\left(f_{1}, f_{2}, f_{3}, f_{4}\right) & =\left(\psi K_{L}, l^{-} X, l^{+} X, \psi K_{S}\right), \\
\text { (II) } \quad\left(f_{1}, f_{2}, f_{3}, f_{4}\right) & =\left(\psi K_{S}, l^{-} X, l^{+} X, \psi K_{L}\right), \\
\text { (III) } \quad\left(f_{1}, f_{2}, f_{3}, f_{4}\right) & =\left(\psi K_{L}, l^{+} X, l^{-} X, \psi K_{S}\right), \\
\text { (IV) } \quad\left(f_{1}, f_{2}, f_{3}, f_{4}\right) & =\left(\psi K_{S}, l^{+} X, l^{-} X, \psi K_{L}\right) .
\end{aligned}
$$

For all the processes which we consider, we can find $\Delta N_{R}, \Delta \sigma, y \Gamma t, \Delta \mathcal{C}, \hat{\mathcal{S}}, \hat{\mathcal{C}}$ are small numbers compared with $\mathcal{O}(1)$. Expanding eq. (2.7) with respect to the small parameters, one obtains the asymmetry at linear order approximation,

$$
\begin{aligned}
A \simeq & R_{T}+C_{T} \cos (x \Gamma t)+S_{T} \sin (x \Gamma t) \\
& +B_{T} \sin ^{2}(x \Gamma t)+D_{T} \sin (x \Gamma t) \cos (x \Gamma t)+E_{T}(y \Gamma t) \sin (x \Gamma t),
\end{aligned}
$$

where,

$$
\begin{aligned}
& R_{T}=-\frac{\Delta N_{R}}{2}+\frac{\Delta \sigma}{2} y \Gamma t \simeq-\frac{\Delta N_{R}}{2}, \\
& C_{T}=\frac{\Delta \mathcal{C}}{2}, \quad S_{T}=\frac{\Delta \mathcal{S}}{2}, \\
& B_{T}=-\frac{\Delta \mathcal{S}}{4} \hat{S}, \quad D_{T}=-\frac{\Delta \mathcal{S}}{4} \hat{C}, \\
& E_{T}=-\frac{\Delta \mathcal{S}}{4} \hat{\sigma} .
\end{aligned}
$$

We ignore $\Delta \sigma y$ in eqs. (4.3)-(4.6). $\hat{\sigma}$ and $\Delta \mathcal{S}$ are $\mathcal{O}(1)$ parameters and $\hat{\sigma} y$ gives rise to small contribution. The model independent parametrization in eq. (4.2) without the last term can be found in [6]. In each process, we compute the asymmetry and the coefficients $\left(R_{T}, C_{T}, S_{T}, B_{T}, D_{T}, E_{T}\right)$. We label suffix I $\sim \mathrm{IV}$ on the quantities corresponding to each process to distinguish them. Below and in table 4, we show the asymmetry and the coefficients for the process I. For the other processes, we show them in tables 5-7. We first investigate $\Delta N_{R}$ in eq. (2.8) for the process I. With eq. (D.4), one obtains,

$$
\Delta N_{R}^{I}=2\left[-S z^{I}+R_{M}+\hat{\lambda}_{\mathrm{wst}}^{R}-G \hat{\lambda}_{l}^{R}-C_{\xi}^{l}-\xi_{l}^{R}\right] .
$$

With eq. (4.7) and eqs. (D.5)-(D.9), one can also derive the coefficients in the asymmetry,

$$
\begin{aligned}
R_{T}^{I} & =-\frac{\Delta N_{R}^{I}}{2}=S z^{I}-R_{M}-\hat{\lambda}_{\mathrm{wst}}^{R}+G \hat{\lambda}_{l}^{R}+C_{\xi}^{l}+\xi_{l}^{R}, \\
C_{T}^{I} & =\frac{\Delta \mathcal{C}^{I}}{2}=C-S z^{I}+\theta_{K}^{R}+S \Delta \lambda_{l}^{I}=C^{\prime}-2 \epsilon_{K}^{R}-S z^{I}+\theta_{K}^{R}+S \Delta \lambda_{l}^{I}, \\
S_{T}^{I} & =\frac{\Delta \mathcal{S}^{I}}{2}=-\left[S\left(1-G z^{R}\right)-G \theta_{K}^{I}+G S \Delta \lambda_{l}^{R}\right], \\
B_{T}^{I} & =-\frac{\Delta \mathcal{S}^{I}}{4} \hat{S}^{I} \simeq \frac{S}{2} \hat{S}^{I} \\
& =S\left[G\left(z_{K}^{I}-\Delta \lambda_{\mathrm{wst}}^{I}\right)-z^{I}+S R_{M}+S \hat{\lambda}_{\mathrm{wst}}^{R}-S C_{\xi}^{l}-S \xi_{l}^{R}\right], \\
D_{T}^{I} & =-\frac{\Delta \mathcal{S}^{I}}{4} \hat{C}^{I} \simeq \frac{S}{2} \hat{C}^{I}=S\left[z_{K}^{R}-\Delta \lambda_{\mathrm{wst}}^{R}-G z^{R}-S \hat{\lambda}_{l}^{I}\right], \\
E_{T}^{I} & =-\frac{\Delta \mathcal{S}^{I}}{4} \hat{\sigma}^{I} \simeq G S .
\end{aligned}
$$


Note that $C^{\prime}$ and $\epsilon_{K}^{R}$ are phase convention independent parameters due to definition of $C^{\prime}$. Therefore, we state that all of eqs. (4.8)-(4.13) are expressed as phase convention independent parameters. In eq. (4.9), effect of indirect $\mathrm{CP}$ violation in $\mathrm{K}$ meson system explicitly appears and gives rise to $\mathcal{O}\left(10^{-3}\right)$ contribution to $C_{T}^{I}$. Assuming $|q / p|-1,\left|\bar{A}_{\psi \bar{K}^{0}} / A_{\psi K^{0}}\right|-1$, $\left|1+\theta_{K}\right|-1$ are small numbers, we can expand $C^{\prime}$ in eq. (3.30) as,

$$
C^{\prime} \simeq 2-\left|\frac{q}{p}\right|-\left|\frac{\bar{A}_{\psi \bar{K}^{0}}}{A_{\psi K^{0}}}\right|-\theta_{K}^{R}, \quad\left|\frac{q}{p}\right| \simeq 1-\frac{1}{2} \operatorname{Im}\left(\frac{\Gamma_{12}^{d}}{M_{12}^{d}}\right) .
$$

A theoretical prediction for the imaginary part of $\Gamma_{12}^{d} / M_{12}^{d}$ is calculated [8], and it shows $\operatorname{Im}\left(\Gamma_{12}^{d} / M_{12}^{d}\right) \sim \mathcal{O}\left(10^{-4}\right)$. Direct $\mathrm{CP}$ violation in $B_{d}^{0} \rightarrow \psi K^{0}$ is $1-\left|\bar{A}_{\psi \bar{K}^{0}} / A_{\psi K^{0}}\right| \simeq$ $\mathcal{O}\left(10^{-3}\right)[9]-[10]$. Hence, $\epsilon_{K}^{R},\left|\bar{A}_{\psi \bar{K}^{0}} / A_{\psi K^{0}}\right| \sim \mathcal{O}\left(10^{-3}\right)$ are dominant in $C_{T}^{I}$, if CPT violations and the wrong sign decay in $B \rightarrow l X$ in eq. (4.9) are also negligible.

If $R_{T}, C_{T}, S_{T}, B_{T}$ and $D_{T}$ were genuine T-odd quantities, they would vanish in the limit of T-symmetry. In other words, if there are non-vanishing contributions in the limit of T-symmetry, $R_{T}, C_{T}, S_{T}, B_{T}$ and $D_{T}$ are not T-odd quantities. From eqs. (4.8)-(4.13), we find the T-even contributions. Some of them do not vanish in the limit of T-symmetry and they include $C_{\xi}^{l}, \hat{\lambda}_{\mathrm{wst}}^{R} \theta_{K}^{R}$, etc. The others are quadratic with respect to T-odd quantities and they vanish in the limit of T-symmetry. They include $S \Delta \lambda_{l}^{I}, S \Delta \lambda_{\text {wst }}^{R}, S^{2} \hat{\lambda}_{l}^{I}$, etc.

Now we study condition that the asymmetry becomes a T-odd quantity. The following equations are needed for T-even terms in each coefficient to vanish,

$$
\begin{aligned}
\hat{\lambda}_{\mathrm{wst}}^{R}=0, \quad G \hat{\lambda}_{l}^{R}=0, \quad C_{\xi}^{l} & =0 \rightarrow R_{T}^{I}: \mathrm{T}-\text { odd }, \\
\theta_{K}^{R}=0, \quad S \Delta \lambda_{l}^{I}=0 & \rightarrow C_{T}^{I}: \mathrm{T}-\text { odd }, \\
G \theta_{K}^{I}=0, \quad G S \Delta \lambda_{l}^{R}=0 & \rightarrow S_{T}^{I}: \mathrm{T}-\text { odd }, \\
S G \Delta \lambda_{\mathrm{wst}}^{I}=0, \quad S^{2} \hat{\lambda}_{\mathrm{wst}}^{R}=0, \quad S^{2} C_{\xi}^{l}=0 & \rightarrow B_{T}^{I}: \mathrm{T}-\text { odd }, \\
S \Delta \lambda_{\mathrm{wst}}^{R}=0, \quad S^{2} \hat{\lambda}_{l}^{I}=0 & \rightarrow D_{T}^{I}: \mathrm{T}-\text { odd } .
\end{aligned}
$$

When the real part and imaginary part of $\lambda$ do not vanish, both $G$ and $S$ are non-zero and the conditions that all the eqs. (4.15)-(4.19) are satisfied become,

$$
\theta_{K}=\Delta \lambda_{\mathrm{wst}}=\Delta \lambda_{l}=\hat{\lambda}_{l}=\hat{\lambda}_{\mathrm{wst}}^{R}=C_{\xi}^{l}=0 .
$$

The conditions except $C_{\xi}^{l}=0$ agree with ones obtained in [6]. The additional condition is required since we take account of the overall constants in the asymmetry.

In the first column of table 4 , we show how each coefficient of the asymmetry in eq. (4.2) depends on T-odd combination of the parameters and in the other columns we show the dependence on T-even combination of the parameters. As for T-even contribution, we identify the sources of T-even contribution to the coefficients. In the second column, the contribution of $\theta_{K}$ which is $\mathrm{CP}$ and $\mathrm{CPT}$ violation in right strangeness decays is shown. In the third column, the contribution of $C_{\xi}^{l}$ which is CP and CPT violation in the right sign semi-leptonic decays is shown. In the fourth and the fifth column, T-even contribution from the wrong strangeness decays and the wrong sign semi-leptonic decays are shown, respectively. In tables $5-7$, we show the coefficients for the processes (II)-(IV). In appendix E, we show a rule useful for deriving them. 


\begin{tabular}{|c|c|c|c|c|c|}
\hline & T-odd terms & $\theta_{K} \neq 0$ & $C_{\xi}^{l} \neq 0$ & $A_{\psi \bar{K}^{0}} \neq 0, \bar{A}_{\psi K^{0}} \neq 0$ & $\bar{A}_{l^{+}} \neq 0, A_{l^{-}} \neq 0$ \\
\hline$R_{T}^{I}$ & $S z^{I}-R_{M}+\xi_{l}^{R}$ & 0 & $C_{\xi}^{l}$ & $-\hat{\lambda}_{\mathrm{wst}}^{R}$ & $G \hat{\lambda}_{l}^{R}$ \\
\hline$C_{T}^{I}$ & $C-S z^{I}$ & $\theta_{K}^{R}$ & 0 & 0 & $S \Delta \lambda_{l}^{I}$ \\
\hline$S_{T}^{I}$ & $-S\left[1-G z^{R}\right]$ & $G \theta_{K}^{I}$ & 0 & 0 & $-G S \Delta \lambda_{l}^{R}$ \\
\hline$B_{T}^{I}$ & $S\left[G z_{K}^{I}-z^{I}+S R_{M}-S \xi_{l}^{R}\right]$ & 0 & $-S^{2} C_{\xi}^{l}$ & $S^{2} \hat{\lambda}_{\mathrm{wst}}^{R}-S G \Delta \lambda_{\mathrm{wst}}^{I}$ & 0 \\
\hline$D_{T}^{I}$ & $S\left[z_{K}^{R}-G z^{R}\right]$ & 0 & 0 & $-S \Delta \lambda_{\mathrm{wst}}^{R}$ & $-S^{2} \hat{\lambda}_{l}^{I}$ \\
\hline$E_{T}^{I}$ & $G S$ & 0 & 0 & 0 & 0 \\
\hline
\end{tabular}

Table 4. The coefficients of the asymmetry for the process I with the final state $\left(f_{1}, f_{2}, f_{3}, f_{4}\right)=$ $\left(\psi K_{L}, l^{-} X, l^{+} X, \psi K_{S}\right)$ and the sources which give rise to the non-vanishing contribution to the asymmetry. The sources of the first column corresponds to T-odd terms and the other correspond to T-even terms.

\begin{tabular}{|c|c|c|c|c|c|}
\hline & T-odd terms & $\theta_{K} \neq 0$ & $C_{\xi}^{l} \neq 0$ & $A_{\psi K^{0}} \neq 0, \bar{A}_{\psi K^{0}} \neq 0$ & $\bar{A}_{l^{+}} \neq 0, A_{l^{-}} \neq 0$ \\
\hline$R_{T}^{I I}$ & $-S z^{I}-R_{M}+\xi_{l}^{R}$ & 0 & $C_{\xi}^{l}$ & $\hat{\lambda}_{\mathrm{wst}}^{R}$ & $-G \hat{\lambda}_{l}^{R}$ \\
\hline$C_{T}^{I I}$ & $C+S z^{I}$ & $\theta_{K}^{R}$ & 0 & 0 & $-S \Delta \lambda_{l}^{I}$ \\
\hline$S_{T}^{I I}$ & $S\left[1+G z^{R}\right]$ & $-G \theta_{K}^{I}$ & 0 & 0 & $-G S \Delta \lambda_{l}^{R}$ \\
\hline$B_{T}^{I I}$ & $-S\left[G z_{K}^{I}-z^{I}-S R_{M}+S \xi_{l}^{R}\right]$ & 0 & $-S^{2} C_{\xi}^{l}$ & $-S^{2} \hat{\lambda}_{\mathrm{wst}}^{R}+S G \Delta \lambda_{\mathrm{wst}}^{I}$ & 0 \\
\hline$D_{T}^{I I}$ & $S\left[z_{K}^{R}-G z^{R}\right]$ & 0 & 0 & $-S \Delta \lambda_{\mathrm{wst}}^{R}$ & $-S^{2} \hat{\lambda}_{l}^{I}$ \\
\hline$E_{T}^{I I}$ & $G S$ & 0 & 0 & 0 & 0 \\
\hline
\end{tabular}

Table 5. The coefficients of the asymmetry for the process II with the final state $\left(f_{1}, f_{2}, f_{3}, f_{4}\right)=$ $\left(\psi K_{S}, l^{-} X, l^{+} X, \psi K_{L}\right)$ and the sources which give rise to the non-vanishing contribution to the asymmetry. The sources of the first column corresponds to T-odd terms and the other correspond to T-even terms.

\begin{tabular}{|c|c|c|c|c|c|}
\hline & T-odd terms & $\theta_{K} \neq 0$ & $C_{\xi}^{l} \neq 0$ & $A_{\psi \bar{K}^{0}} \neq 0, \bar{A}_{\psi K^{0}} \neq 0$ & $\bar{A}_{l^{+}} \neq 0, A_{l^{-}} \neq 0$ \\
\hline$R_{T}^{I I I}$ & $S z^{I}+R_{M}-\xi_{l}^{R}$ & 0 & $-C_{\xi}^{l}$ & $-\hat{\lambda}_{\mathrm{wst}}^{R}$ & $G \hat{\lambda}_{l}^{R}$ \\
\hline$C_{T}^{I I I}$ & $-C-S z^{I}$ & $-\theta_{K}^{R}$ & 0 & 0 & $S \Delta \lambda_{l}^{I}$ \\
\hline$S_{T}^{I I I}$ & $S\left[1+G z^{R}\right]$ & $-G \theta_{K}^{I}$ & 0 & 0 & $-G S \Delta \lambda_{l}^{R}$ \\
\hline$B_{T}^{I I I}$ & $S\left[G z_{K}^{I}-z^{I}-S R_{M}+S \xi_{l}^{R}\right]$ & 0 & $S^{2} C_{\xi}^{l}$ & $S^{2} \hat{\lambda}_{\mathrm{wst}}^{R}-S G \Delta \lambda_{\mathrm{wst}}^{I}$ & 0 \\
\hline$D_{T}^{I I I}$ & $S\left[z_{K}^{R}-G z^{R}\right]$ & 0 & 0 & $-S \Delta \lambda_{\mathrm{wst}}^{R}$ & $-S^{2} \hat{\lambda}_{l}^{I}$ \\
\hline$E_{T}^{I I I}$ & $-G S$ & 0 & 0 & 0 & 0 \\
\hline
\end{tabular}

Table 6. The coefficients of the asymmetry for the process III with the final states $\left(f_{1}, f_{2}, f_{3}, f_{4}\right)=$ $\left(\psi K_{L}, l^{+} X, l^{-} X, \psi K_{S}\right)$ and the sources which give rise to the non-vanishing contribution to the asymmetry. The sources of the first column corresponds to T-odd terms and the other correspond to T-even terms. 


\begin{tabular}{|l|c|c|c|c|c|}
\hline & T-odd terms & $\theta_{K} \neq 0$ & $C_{\xi}^{l} \neq 0$ & $A_{\psi K^{0}} \neq 0, \bar{A}_{\psi K^{0}} \neq 0$ & $\bar{A}_{l^{+}} \neq 0, A_{l^{-}} \neq 0$ \\
\hline$R_{T}^{I V}$ & $-S z^{I}+R_{M}-\xi_{l}^{R}$ & 0 & $-C_{\xi}^{l}$ & $\hat{\lambda}_{\mathrm{wst}}^{R}$ & $-G \hat{\lambda}_{l}^{R}$ \\
\hline$C_{T}^{I V}$ & $-C+S z^{I}$ & $-\theta_{K}^{R}$ & 0 & 0 & $-S \Delta \lambda_{l}^{I}$ \\
\hline$S_{T}^{I V}$ & $-S\left[1-G z^{R}\right]$ & $G \theta_{K}^{I}$ & 0 & 0 & $-G S \Delta \lambda_{l}^{R}$ \\
\hline$B_{T}^{I V}$ & $S\left[-G z_{K}^{I}+z^{I}-S R_{M}+S \xi_{l}^{R}\right]$ & 0 & $S^{2} C_{\xi}^{l}$ & $-S^{2} \hat{\lambda}_{\mathrm{wst}}^{R}+S G \Delta \lambda_{\mathrm{wst}}^{I}$ & 0 \\
\hline$D_{T}^{I V}$ & $S\left[z_{K}^{R}-G z^{R}\right]$ & 0 & 0 & $-S \Delta \lambda_{\mathrm{wst}}^{R}$ & $-S^{2} \hat{\lambda}_{l}^{I}$ \\
\hline$E_{T}^{I V}$ & $-G S$ & 0 & 0 & 0 & 0 \\
\hline
\end{tabular}

Table 7. The coefficients of the asymmetry for the process IV with the final states $\left(f_{1}, f_{2}, f_{3}, f_{4}\right)=$ $\left(\psi K_{S}, l^{+} X, l^{-} X, \psi K_{L}\right)$ and the sources which give rise to the non-vanishing contribution to the asymmetry. The sources of the first column corresponds to T-odd terms and the other correspond to T-even terms.

Although the asymmetry in eq. (4.2) is not exactly T-asymmetry, the measurement of the coefficients are useful for constraining $S$ and $G$ as well as various non-standard interactions. Non-standard interactions include wrong sign decay and CPT violation. In the following subsections, we show how one can determine $S$ and $G$ and also show how one can constrain the various non-standard interactions. We first study the case without any assumption and in later subsections, we investigate two physically interesting cases, one corresponding to the case that CPT is a good symmetry and the other is the case without wrong sign decays. Since there are relations among the coefficients for different processes, we first identify the independent coefficients. From tables $4-7$, one finds the following relations among the coefficients for the different processes.

$$
\begin{array}{rlrl}
R_{T}^{I V} & =-R_{T}^{I}, & R_{T}^{I I I} & =-R_{T}^{I I}, \\
C_{T}^{I I I} & =-C_{T}^{I I}, & C_{T}^{I V} & =-C_{T}^{I}, \\
S_{T}^{I I I} & =S_{T}^{I I}, & & S_{T}^{I V}=S_{T}^{I}, \\
B_{T}^{I I I} & =-B_{T}^{I I}, & & B_{T}^{I V}=-B_{T}^{I}, \\
D_{T}^{I} & =D_{T}^{I I}=D_{T}^{I I I}=D_{T}^{I V}, & & \\
E_{T}^{I} & =E_{T}^{I I}=-E_{T}^{I I I}=-E_{T}^{I V} . &
\end{array}
$$

They imply that there are ten independent coefficients. In table 8 , we show how ten independent combination of the coefficients can be written in terms of CPT even, CPT odd, and wrong sign decay parameters. Since there are eighteen parameters, the number of the independent coefficients is not enough to extract the parameters. However, one can still constrain the combination of the parameters. Below we investigate how to extract the parameters for the three cases.

\subsection{Extracting the parameters from the coefficients: general case}

Let us first examine how one can determine the parameters from the measurements of the coefficients shown in table 8. Hereafter, we discuss a method to determine the values for $G$ and $S$ through observing $E_{T}$. Since $E_{T}$ is multiplied by $y$ in eq. (4.2), one cannot extract 


\begin{tabular}{|c|c|c|c|}
\hline & CPT even parameters & CPT violating parameters & wrong sign decays \\
\hline$\frac{R_{T}^{I}+R_{T}^{I I}}{2}$ & $-R_{M}+\xi_{l}^{R}$ & $+C_{\xi}^{l}$ & 0 \\
\hline$\frac{R_{T}^{I}-R_{T}^{I I}}{2}$ & 0 & $S z^{I}$ & $-\hat{\lambda}_{\mathrm{wst}}^{R}+G \hat{\lambda}_{l}^{R}$ \\
\hline$\frac{C_{T}^{I}+C_{T}^{I I}}{2}$ & $C$ & $\theta_{K}^{R}$ & 0 \\
\hline$\frac{C_{T}^{I}-C_{T}^{I I}}{2}$ & 0 & $-S z_{I}$ & $S \Delta \lambda_{l}^{I}$ \\
\hline$\frac{S_{T}^{I}+S_{T}^{I I}}{2}$ & 0 & $S G z^{R}$ & $-S G \Delta \lambda_{l}^{R}$ \\
\hline$\frac{S_{T}^{I}-S_{T}^{I I}}{2}$ & $-S$ & $G \theta_{K}^{I}$ & 0 \\
\hline$\frac{B_{T}^{I}+B_{T}^{I I}}{2}$ & $S^{2}\left(R_{M}-\xi_{l}^{R}\right)$ & $-S^{2} C_{\xi}^{l}$ & 0 \\
\hline$\frac{B_{T}^{I}-B_{T}^{I I}}{2}$ & 0 & $S\left(G z_{K}^{I}-z^{I}\right)$ & $S\left(S \hat{\lambda}_{w s t}^{R}-G \Delta \lambda_{w s t}^{I}\right)$ \\
\hline$D_{T}^{I}$ & 0 & $S\left(z_{K}^{R}-G z^{R}\right)$ & $-S\left(\Delta \lambda_{w s t}^{R}+S \hat{\lambda}_{l}^{I}\right)$ \\
\hline$E_{T}^{I}$ & $G S$ & 0 & 0 \\
\hline$\frac{B_{T}^{I}+B_{T}^{I I}}{R_{T}^{I}+R_{T}^{I I}}$ & $-S^{2}$ & 0 & 0 \\
\hline
\end{tabular}

Table 8. Combinations of the independent coefficients in the asymmetry. The sources which contribute to each combination are classified in three categories.

the value of $E_{T}$ solely from the measurement of the asymmetry. Therefore, we need to determine the value of $y$ through the other experiment. $y$ defined in eq. (2.2) is regarding to the width difference of $\mathrm{B}$ meson mass eigenstate, and a method to measure $y \cos \beta \simeq G y$ is suggested in refs. [11]-[12]. Combining the measurement of $E_{T}^{I} y \simeq G S y$, one can determine $S$. Since $S$ and $G$ in their leading order satisfy $S^{2}+G^{2} \simeq 1-\mathcal{O}\left(C^{2}\right)$, the measurement of $E_{T}$ determines $( \pm G, S)$ within the two-fold ambiguity. The ambiguity would be removed if we assume that the standard model contribution is dominant for the width difference. (See figure 1) As an alternative way to determine $S$, one can use the relation $\frac{B_{T}^{I}+B_{T}^{I I}}{R_{T}^{I}+R_{T}^{I I}}=-S^{2}$, and determine $|S|$. The sign ambiguity for $S$ can be removed because in the leading order $S$ is equal to $\frac{S_{T}^{I I}-S_{T}^{I}}{2}$. Excluding the case that the sub-leading contribution changes the sign of the leading term, one can determine the sign for $S$ through observing $\frac{S_{T}^{I I}-S_{T}^{I}}{2}$. Having determined $G$ and $S$, we consider constraining the other parameters.

We note that the following relation is satisfied,

$$
\frac{R_{T}^{I}-R_{T}^{I I}}{2}+\frac{C_{T}^{I}-C_{T}^{I I}}{2}=-\hat{\lambda}_{\mathrm{wst}}^{R}+G \hat{\lambda}_{l}^{R}+S \Delta \lambda_{l}^{I} .
$$

Since the right-hand side is independent of CPT violating parameters, non-vanishing contribution implies the unambiguous evidence of the presence of the wrong sign decay. Furthermore, since $S$ is determined, one can write the CPT violating parameter $\theta_{K}^{I}$ as,

$$
\theta_{K}^{I}=\frac{\frac{S_{T}^{I}-S_{T}^{I I}}{2}+S}{G}
$$




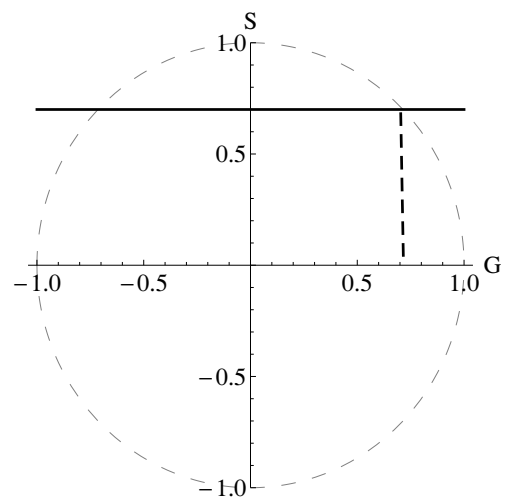

Figure 1. Determination of $G$ and $S$. Due to $G^{2}+S^{2} \simeq 1, G$ and $S$ are on the circle of unit length. Once $S$ is known, $G$ is determined within two-fold ambiguity.

If the right-hand side is non-zero, it implies $\mathrm{CPT}$ violation in the right strangeness decay. However, the real part of $\theta_{K}$ cannot be singly extracted, since

$$
\theta_{K}^{R}+C=\frac{C_{T}^{I}+C_{T}^{I I}}{2} .
$$

One also notes the relation,

$$
-R_{M}+\xi_{l}^{R}+C_{\xi}^{l}=\frac{R_{T}^{I}+R_{T}^{I I}}{2}
$$

If any one of the combinations, $\frac{R_{T}^{I}-R_{T}^{I I}}{2}, \frac{C_{T}^{I}-C_{T}^{I I}}{2}, \frac{S_{T}^{I}+S_{T}^{I I}}{2}, \frac{B_{T}^{I}-B_{T}^{I I}}{2}$, and $D_{T}^{I}$ is non-zero, it implies CPT violation and/or wrong sign decay. However, if the cancellation between CPT violation and wrong sign decay occurs, they can vanish.

\subsection{Extracting the parameters from the coefficients: CPT conserving limit}

Next we consider the case in the limit of CPT symmetry. In the limit of CPT symmetry, all the contribution in the second column vanishes in table 8 . Since all the wrong sign decay parameters are CPT even, the third column of table 8 does not vanish. In the limit $C, S$ and $R_{M}-\xi_{l}^{R}$ can be determined as,

$$
\begin{aligned}
C & =\frac{C_{T}^{I}+C_{T}^{I I}}{2}, \\
S & =\frac{S_{T}^{I I}-S_{T}^{I}}{2}, \\
R_{M}-\xi_{l}^{R} & =-\frac{R_{T}^{I}+R_{T}^{I I}}{2} .
\end{aligned}
$$

Moreover T-odd wrong sign semi-leptonic decay $\Delta \lambda_{l}$ can be determine as,

$$
\begin{aligned}
\Delta \lambda_{l}^{I} & =\frac{C_{T}^{I}-C_{T}^{I I}}{2 S}, \\
\Delta \lambda_{l}^{R} & =-\frac{S_{T}^{I}+S_{T}^{I I}}{2 G S} .
\end{aligned}
$$


For the other five wrong sign decay parameters $\hat{\lambda}_{\text {wst }}^{R}, \hat{\lambda}_{l}^{R, I} \Delta \lambda_{\text {wst }}^{R, I}$, one obtains the following three constraints.

$$
\begin{aligned}
\frac{R_{T}^{I}-R_{T}^{I I}}{2} & =-\hat{\lambda}_{\mathrm{wst}}^{R}+G \hat{\lambda}_{l}^{R}, \\
\frac{B_{T}^{I}-B_{T}^{I I}}{2} & =S\left(S \hat{\lambda}_{\mathrm{wst}}^{R}-G \Delta \lambda_{\mathrm{wst}}^{I}\right), \\
D_{T}^{I} & =-S\left(\Delta \lambda_{\mathrm{wst}}^{R}+S \hat{\lambda}_{l}^{I}\right) .
\end{aligned}
$$

\subsection{Extracting the parameters from the coefficients: case without wrong sign decay}

Lastly, we consider the case without wrong sign decays. The relations in eqs. (4.22)-(4.24) are satisfied in this case. The right-hand side of eq. (4.21) vanishes. In addition to these, $\mathrm{CP}$ and $\mathrm{CPT}$ violation of the mixing parameters in $\mathrm{B}$ meson system is determined by

$$
z^{I}=\frac{R_{T}^{I}-R_{T}^{I I}}{2 S}, \quad z^{R}=\frac{S_{T}^{I}+S_{T}^{I I}}{2 G S} .
$$

$\mathrm{CP}$ and $\mathrm{CPT}$ violation in the neutral $\mathrm{K}$ meson system is also determined as,

$$
z_{K}^{I}=\frac{D_{T}^{I}+\frac{S_{T}^{I}+S_{T}^{I I}}{2}}{S}, \quad z_{K}^{R}=\frac{B_{T}^{I}-B_{T}^{I I}-\left(C_{T}^{I}-C_{T}^{I I}\right)}{2 S G} .
$$

The five parameters $C_{\xi}^{l}, \theta_{K}^{R}, C, R_{M}$ and $\xi_{l}^{R}$ satisfy the two constraints eqs. (4.23)-(4.24).

\section{Conditions for authentic time reversed process}

In section 4, we showed the expression of the asymmetry that describes event number difference of figure 2 and figure 3. However, rather than figure 3, figure 4 is an authentic time reversed process of figure 2, since the two processes of figure 2 and figure 4 are related with flipping time direction. In discussion given in refs. [1]-[3], one substituted figure 3 for figure 4 because signal sides of figure 2 and figure 3 are deemed to be a time reversed process to each other. Since figure 3 is not an authentic time reversed process, the asymmetry is slightly deviated from T-odd.

In this section, we clarify why T-even parts are included in the coefficients eqs. (4.8)(4.12), although it is naively thought to be a T-odd quantity.

One can show that, when the following conditions are simultaneously satisfied, figure 3 plays the role as a time reversed process of figure 2 and the coefficients eqs. (4.8)-(4.12) become T-odd.

1. Equivalence conditions of B meson states.

2. $\Delta N_{R}^{\mathrm{e}}=0$.

Here we denote $\Delta N_{R}=\Delta N_{R}^{\mathrm{o}}+\Delta N_{R}^{\mathrm{e}}$ and $\Delta N_{R}^{\mathrm{e}}\left(\Delta N_{R}^{\mathrm{o}}\right)$ is the T-even (odd) part. 
The equivalence conditions indicate that the initial (final) B meson states of signal side in figures 3-4 are the same as each other. The equivalence conditions are described as,

$$
\left\{\begin{array}{l}
\left|B_{\left(\rightarrow l^{+} X\right) \perp}\right\rangle \propto\left|B_{l-X \rightarrow}\right\rangle \\
\left|B_{\rightarrow \psi K_{S}}\right\rangle \propto\left|B_{\left(\psi K_{L} \rightarrow\right) \perp}\right\rangle
\end{array}\right.
$$

Eq. (5.1) shows that B meson states in figures $3-4$ are equivalent. Similarly, figure 5 is the authentic time reversed process of figure 3 . When we apply the same condition to B meson states in figures $2-5$, one obtains,

$$
\left\{\begin{array}{l}
\left|B_{\left(\rightarrow l^{-} X\right) \perp}\right\rangle \propto\left|B_{\left(l^{+} X \rightarrow\right)}\right\rangle \\
\left.\left|B_{\left(\rightarrow \psi K_{L}\right)}\right\rangle \propto \mid B_{\left(\psi K_{S} \rightarrow\right.}\right) \perp
\end{array} .\right.
$$

Violation of the conditions (5.1)-(5.2) is originally calculated in ref. [6]. Including overall factors and using our notation, we show the violation of the conditions (5.1)-(5.2) as follows,

$$
\begin{gathered}
\left\{\begin{array}{l}
\left\langle B_{\left(\psi K_{L} \rightarrow\right) \perp} \mid B_{\left(\rightarrow \psi K_{S}\right) \perp}\right\rangle=N_{\left(\rightarrow \psi K_{S}\right) \perp} N_{\left(\psi K_{L} \rightarrow\right) \perp}\left(A_{\psi K^{0}} A_{\psi K^{0}}^{\mathrm{ID}}+\bar{A}_{\psi \bar{K}^{0}} \bar{A}_{\psi \bar{K}^{0}}^{\mathrm{ID}}\right) \frac{\theta_{K}+\Delta \lambda_{\mathrm{wst}}}{2} \\
\left\langle B_{\left(l^{-} X \rightarrow\right) \perp} \mid B_{\left(\rightarrow l^{+} X\right) \perp}\right\rangle=2 N_{\left(l^{-} \rightarrow\right) \perp} N_{\left(\rightarrow l^{+}\right) \perp} A_{l^{+}} \bar{A}_{l^{-}}^{\mathrm{ID}} \frac{p}{q} \lambda_{l^{+}},
\end{array}\right. \\
\left\{\begin{array}{l}
\left\langle B_{\left(\psi K_{S} \rightarrow\right) \perp} \mid B_{\left(\rightarrow \psi K_{L}\right) \perp}\right\rangle=N_{\left(\rightarrow \psi K_{L}\right) \perp} N_{\left(\psi K_{S} \rightarrow\right) \perp}\left(A_{\psi K^{0}} A_{\psi K^{0}}^{\mathrm{ID}}+\bar{A}_{\psi \bar{K}^{0}} \bar{A}_{\psi \bar{K}^{0}}^{\mathrm{ID}}\right) \frac{\theta_{K}-\Delta \lambda_{\mathrm{wst}}}{2} \\
\left\langle B_{\left(l^{+} X \rightarrow\right) \perp} \mid B_{\left(\rightarrow l^{-} X\right) \perp}\right\rangle=2 N_{\left(l^{+} \rightarrow\right) \perp} N_{\left(\rightarrow l^{-}\right) \perp} \bar{A}_{l^{-}} A_{l^{+}}^{\mathrm{ID}} \frac{q}{p} \lambda_{l^{-}}^{-1},
\end{array}\right.
\end{gathered}
$$

where we used the expression for states defined in eqs. (F.1)-(F.4), (F.6)-(F.9). In eqs. (5.3)(5.4), effect of mixing-induced $\mathrm{CP}$ violation in $\mathrm{K}$ meson system is included in terms of our notation $\Delta \lambda_{\text {wst }} \simeq \frac{p_{K}}{q_{K}} \frac{A_{\psi \bar{K}^{0}}}{A_{\psi K^{0}}}-\frac{q_{K}}{p_{K}} \frac{\bar{A}_{\psi K^{0}}}{\bar{A}_{\psi \bar{K}^{0}}}$ in comparison with ref. [6].

$\left\langle B_{\left(l^{-} X \rightarrow\right) \perp} \mid B_{\left(\rightarrow l^{+} X\right) \perp}\right\rangle \neq 0$ and $\left\langle B_{\left(l^{+} X \rightarrow\right) \perp} \mid B_{\left(\rightarrow l^{-} X\right) \perp}\right\rangle \neq 0$ indicate that one cannot exactly conduct the flavor tagging in the presence of wrong sign semi-leptonic decays. Similarly, $\left\langle B_{\left(\rightarrow \psi K_{S}\right) \perp} \mid B_{\left(\psi K_{L} \rightarrow\right) \perp}\right\rangle \neq 0$ and $\left\langle B_{\left(\psi K_{S} \rightarrow\right) \perp} \mid B_{\left(\rightarrow \psi K_{L}\right) \perp}\right\rangle \neq 0$ imply that one cannot exactly carry out the $\mathrm{CP}$ tagging in the presence of CPT violation in decays and wrong sign strangeness decays. Therefore, eqs. (5.3)-(5.4) describe that semi-leptonic decays and strangeness changing decays yield tagging ambiguities, and that are expressed in terms of state non-orthogonality.

Then, we turn to explanation of the second condition, $\Delta N_{R}^{\mathrm{e}}=0$. We define the following quantities for the expedient sake.

$$
X^{\mathrm{o}}=\frac{X_{\left(\psi K_{L}\right) \perp, l^{+} X}}{\kappa_{\left(\psi K_{L}\right) \perp, l^{+} X}}-\frac{X_{\left(l^{-} X\right) \perp, \psi K_{S}}}{\kappa_{\left(l^{-} X\right) \perp, \psi K_{S}}}, \quad X^{\mathrm{e}}=\frac{X_{\left(\psi K_{L}\right) \perp, l^{+} X}}{\kappa_{\left(\psi K_{L}\right) \perp, l^{+} X}}+\frac{X_{\left(l^{-} X\right) \perp, \psi K_{S}}}{\kappa_{\left(l^{-} X\right) \perp, \psi K_{S}}},
$$

where $X=\sigma, \mathcal{C}$ and $\mathcal{S}$ are given in eqs. (A.3)-(A.5). Consider the case that the equivalence conditions are satisfied to demonstrate that violation of $\Delta N_{R}^{\mathrm{e}}=0$ gives rise to T-even 


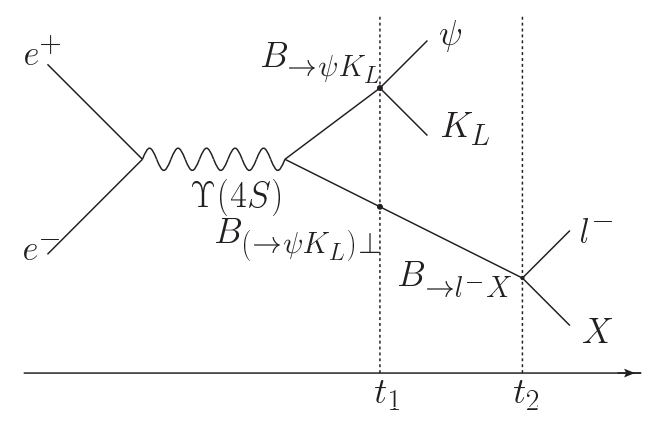

Figure 2. A process with $\left(f_{1}, f_{2}\right)=\left(\psi K_{L}, l^{-} X\right)$.

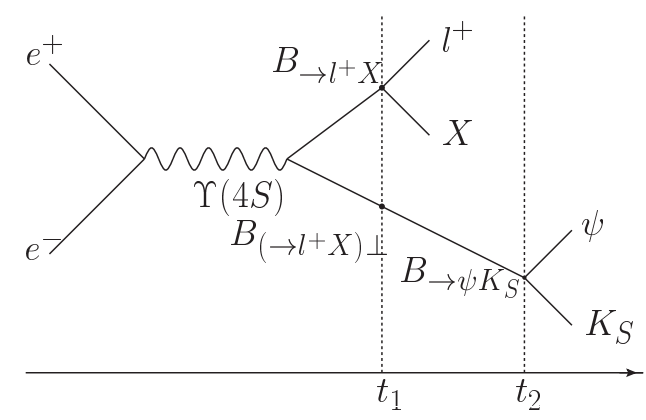

Figure 3. A process with $\left(f_{3}, f_{4}\right)=\left(l^{+} X, \psi K_{S}\right)$. Figure 2 and figure 3 are referred as (I) in eq. (4.1). Event number asymmetry of figure 2 and figure 3. is calculated as eqs. (4.8)-(4.13).

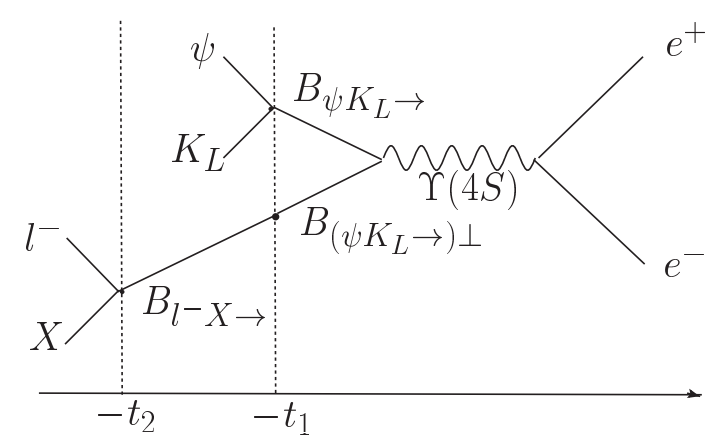

Figure 4. A process with inverse decays of B meson. Figure 2 and figure 4 are related with flipping time direction.

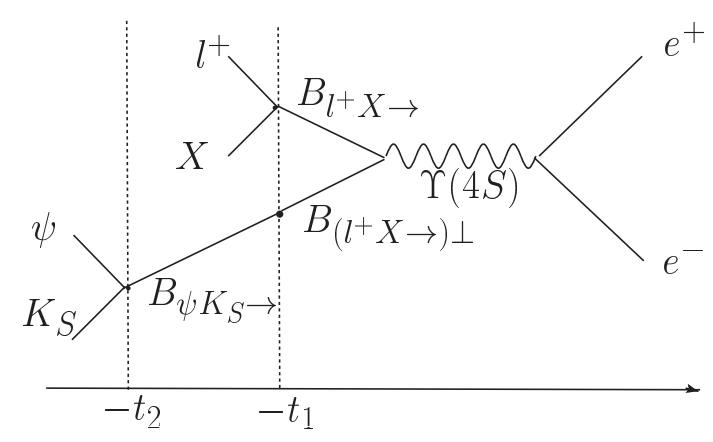

Figure 5. A process with inverse decays of B meson. Figure 3 and figure 5 are related with flipping time direction. 
contribution to the asymmetry. For that case, we can find that $X^{\mathrm{o}}\left(X^{\mathrm{e}}\right)$ defined in eq. (5.5) is T-odd (even) due to expressions given as follows,

$$
\begin{aligned}
& S^{\mathrm{o}}=-2 S\left(1-G z^{R}\right), \quad C^{\mathrm{o}}=2\left[C-S z^{I}\right], \quad\left(\sigma^{\mathrm{o}}\right)^{l}=0, \\
& S^{\mathrm{e}}=2\left[G z_{K}^{I}+\left(S^{2}-1\right) z^{I}\right], \quad C^{\mathrm{e}}=2\left[z_{K}^{R}-G z^{R}\right], \quad\left(\sigma^{\mathrm{e}}\right)^{l}=2 G,
\end{aligned}
$$

where for $\Delta \sigma$ and $\hat{\sigma}$, we write down only the leading part since small parts of $\Delta \sigma$ and $\hat{\sigma}$ are neglected when multiplied by $y$ in the asymmetry (2.7). For the process (I), $\Delta X$ and $\hat{X}$ defined in eqs. $(2.5)-(2.6)$ are written as,

$$
\begin{gathered}
\Delta X \simeq X^{\mathrm{o}}-\frac{\Delta N_{R}}{2} X^{\mathrm{e}}=\left(X^{\mathrm{o}}-\frac{\Delta N_{R}^{\mathrm{o}}}{2} X^{\mathrm{e}}\right)-\frac{\Delta N_{R}^{\mathrm{e}}}{2} X^{\mathrm{e}}, \\
\hat{X} \simeq X^{\mathrm{e}}-\frac{\Delta N_{R}}{2} X^{\mathrm{o}}=\left(X^{\mathrm{e}}-\frac{\Delta N_{R}^{\mathrm{o}}}{2} X^{\mathrm{o}}\right)-\frac{\Delta N_{R}^{\mathrm{e}}}{2} X^{\mathrm{o}} .
\end{gathered}
$$

One finds that the T-even part of $\Delta N_{R}$ leads T-even contribution to $\Delta X$ in eq. (5.8). The same applies to $\hat{X}$, and it is shown that $\Delta X(\hat{X})$ deviates from T-odd (even) when $\Delta N_{R}^{\mathrm{e}}$ has non-zero value. Therefore, we can demonstrate that $\Delta N_{R}^{\mathrm{e}}$ gives rise to T-even contribution to the asymmetry given in eqs. (4.3)-(4.6).

\section{Conclusion}

In this paper, the precise meaning of the time reversal-like asymmetry is investigated, based on the most general time dependence of the asymmetry in eq. (4.2). In analysis of BaBar [4] and [6], the difference of the overall constants for the rates is eliminated. The ratio of the overall constants for the two decay rates is deviated from unity, and the deviation $\Delta N_{R}=N_{R}-1$ is taken into account in our analysis. If one takes the limits $\Delta N_{R}=0$ and $y=0$ in our analysis, the asymmetry of BaBar collaboration [4] is obtained. In our analysis, since the final states $\psi K_{S, L}$ are not the exact CP eigenstates, one can find the effect of mixing-induced CP violation in $\mathrm{K}$ meson system. The effect of $\epsilon_{K}$ is extracted and it gives rise to $\mathcal{O}\left(10^{-3}\right)$ contribution to $C_{T}$, the coefficient of $\cos (x \Gamma t) \cdot \epsilon_{K}^{R}$ and direct CP violation $\left|\bar{A}_{\psi \bar{K}^{0}} / A_{\psi K^{0}}\right|$ are dominant in $C_{T}$, if the wrong sign semi-leptonic decay and CPT violations are negligible. As well as $\epsilon_{K}$, the contribution from CPT violation in Kaon system $z_{K}$ is estimated.

We introduced the parameters which have the specific property under $\mathrm{CP}, \mathrm{T}$ and $\mathrm{CPT}$ transformations, including the effect of indirect $\mathrm{CP}$ violation in $\mathrm{K}$ meson system. Taking account of the difference for overall constants, the coefficients of each time-dependent function are written in terms of such parameters, and one can find that the asymmetry consists of not only T-odd terms, but also T-even terms in the most general time dependent function for the asymmetry. Furthermore, the introduced parameters are invariant under rephasing of quarks. We also found that the asymmetry is expressed as phase convention independent quantities.

We obtained the coefficients of the asymmetry for the processes (I-IV) and studied how to extract the parameters. Assuming that the value of $y$, i.e., the width difference of $B_{d}$ 
meson mass eigenstates is known, the three cases to constrain the parameters are discussed. For the most general case, combining the coefficients for different processes enables one to determine the parameters, $S$ and $G$. We also find that non-zero value of some combination of the coefficient signals either CPT violation or the presence of the wrong sign decays. The other two cases correspond to CPT-conserving limit and the absence of wrong sign decays. In the CPT-conserving case, the coefficients constrain the parameters for wrong sign decays. In the absence of wrong sign decays, indirect CPT violation for B meson and $\mathrm{K}$ meson is constrained.

Moreover, we discussed T-even parts in the asymmetry. We found that T-even terms in the asymmetry vanish when several conditions are satisfied. These derived conditions are categorized as two parts. The first one is referred as equivalence conditions, regarding to B meson states for a time reversal-like process and an authentic time reversed process. As suggested in [6], B mesons for the two processes are not equivalent to each other, and we also showed the violation of the equivalence conditions, including the effects of mixing in $\mathrm{K}$ meson system. Since non-zero $\Delta N_{R}$ is taken into account in our study, $\Delta N_{R}$ can be the origin of T-even contribution to the asymmetry. We investigated that the second condition, which requires that T-even parts of $\Delta N_{R}$ are zero, is needed for the asymmetry to become a T-odd quantity.

\section{A Coefficients of master formula}

We record coefficients of the master formula for the time-dependent decay rate of ref. [6].

$$
\begin{aligned}
N_{(i) \perp, j}= & \frac{1}{4} \mathcal{N}_{i} \mathcal{N}_{j}\left\{1+\left(C_{i}+C_{j}\right)\left(R_{M}-z^{R}\right)\right\}, \quad \mathcal{N}_{i}=\left|A_{i}\right|^{2}+\left|\bar{A}_{i}\right|^{2}, \\
\kappa_{(i) \perp, j}= & \left(1-G_{i} G_{j}\right) \\
& +\left[\left(C_{i}+C_{j}\right)\left(1-G_{i} G_{j}\right)+C_{j} G_{i}+C_{i} G_{j}\right] z^{R}-\left(S_{i}+S_{j}\right) z^{I} \\
& +G_{i} G_{j}\left(C_{i} \theta_{i}^{R}+C_{j} \theta_{j}^{R}\right)-G_{i} S_{j} \theta_{j}^{I}-G_{j} S_{i} \theta_{i}^{I}, \\
\sigma_{(i) \perp, j}= & G_{j}-G_{i} \\
& +\left[C_{i}\left(1+G_{j}-G_{i}\right)-C_{j}\left(1-G_{j}+G_{i}\right)\right] z^{R}+\left(G_{i} S_{j}-G_{j} S_{i}\right) z^{I} \\
& -C_{j} G_{j} \theta_{j}^{R}+S_{j} \theta_{j}^{I}+C_{i} G_{i} \theta_{i}^{R}-S_{i} \theta_{i}^{I}, \\
\mathcal{C}_{(i) \perp, j}= & -C_{i} C_{j}-S_{i} S_{j} \\
& -\left[\left(C_{i}+C_{j}\right)\left(C_{i} C_{j}+S_{i} S_{j}\right)+C_{i} G_{j}+C_{j} G_{i}\right] z^{R}+\left(S_{i}+S_{j}\right) z^{I} \\
& +G_{j} S_{i} \theta_{j}^{I}-\left[C_{i}\left(1-C_{j}^{2}\right)-C_{j} S_{i} S_{j}\right] \theta_{j}^{R} \\
& +G_{i} S_{j} \theta_{i}^{I}-\left[C_{j}\left(1-C_{i}^{2}\right)-C_{i} S_{i} S_{j}\right] \theta_{i}^{R}, \\
\mathcal{S}_{(i) \perp, j}= & C_{i} S_{j}-C_{j} S_{i} \\
& +\left[C_{i} C_{j}\left(S_{j}-S_{i}\right)-\left(C_{j}^{2}+G_{j}\right) S_{i}+\left(C_{i}^{2}+G_{i}\right) S_{j}\right] z^{R}+\left(C_{j}-C_{i}\right) z^{I} \\
& -C_{i} G_{j} \theta_{j}^{I}+\left[\left(C_{j}^{2}-1\right) S_{i}-C_{i} C_{j} S_{j}\right] \theta_{j}^{R} \\
& +C_{j} G_{i} \theta_{i}^{I}-\left[\left(C_{i}^{2}-1\right) S_{j}-C_{i} C_{j} S_{i}\right] \theta_{i}^{R},
\end{aligned}
$$

where $A_{i}$ and $\bar{A}_{i}$ in eq. (A.1) are defined in eq. (3.1). $i$ and $j$ represent the final state of tagging side $\left(f_{i}\right)$ and signal side $\left(f_{j}\right)$ for a pair of $\mathrm{B}$ meson decaying respectively. 


\section{B Incoming mass eigenstates and outgoing mass eigenstates in B meson and $\mathrm{K}$ meson system}

Throughout this paper, the time reversal process of B meson decay often appears. To describe the inverse decay amplitudes, as out-states of B mesons, the reciprocal base must be used for Non-Hermitian Hamiltonian system. This is formulated in several literatures, refs. [13]-[17]. In this appendix, we show the definition of incoming states and outgoing states which are used in this paper.

The incoming mass eigenstates of effective Hamiltonian in B meson system are

$$
\begin{aligned}
& \left|B_{H}^{\mathrm{in}}\right\rangle=p \sqrt{1+z}\left|B^{0}\right\rangle-q \sqrt{1-z}\left|\bar{B}^{0}\right\rangle, \\
& \left|B_{L}^{\mathrm{in}}\right\rangle=p \sqrt{1-z}\left|B^{0}\right\rangle+q \sqrt{1+z}\left|\bar{B}^{0}\right\rangle,
\end{aligned}
$$

where $p, q$ are mixing parameters in indirect $\mathrm{CP}$ violation and $z$ is a mixing parameter in indirect $\mathrm{CP}, \mathrm{CPT}$ violation. In terms of matrix elements of effective Hamiltonian, these parameters are written as

$$
\begin{aligned}
\frac{p}{q} & =\sqrt{\frac{M_{12}-\frac{i}{2} \Gamma_{12}}{M_{12}^{*}-\frac{i}{2} \Gamma_{12}^{*}}}, \\
z & =\frac{M_{11}-M_{22}-\frac{i}{2}\left(\Gamma_{11}-\Gamma_{22}\right)}{m_{L}-m_{H}-\frac{i}{2}\left(\Gamma_{L}-\Gamma_{H}\right)} .
\end{aligned}
$$

These expressions help us understand the transformation property of $\lambda, S, G, C$, etc. Then, outgoing mass states are determined to fulfill the following conditions,

$$
\left\langle B_{H}^{\text {out }} \mid B_{H}^{\text {in }}\right\rangle=1,\left\langle B_{H}^{\text {out }} \mid B_{L}^{\text {in }}\right\rangle=0,\left\langle B_{L}^{\text {out }} \mid B_{L}^{\text {in }}\right\rangle=1,\left\langle B_{L}^{\text {out }} \mid B_{H}^{\text {in }}\right\rangle=0 .
$$

Thus, outgoing mass eigenstates are

$$
\begin{aligned}
\left\langle B_{H}^{\text {out }}\right| & =\frac{1}{2 p q}\left(q \sqrt{1+z}\left\langle B^{0}\right|-p \sqrt{1-z}\left\langle\bar{B}^{0}\right|\right), \\
\left\langle B_{L}^{\text {out }}\right| & =\frac{1}{2 p q}\left(q \sqrt{1-z}\left\langle B^{0}\right|+p \sqrt{1+z}\left\langle\bar{B}^{0}\right|\right) .
\end{aligned}
$$

For K meson system, similarly,

$$
\begin{aligned}
\left|K_{L}^{\text {in }}\right\rangle & =p_{K} \sqrt{1+z_{K}}\left|K^{0}\right\rangle-q_{K} \sqrt{1-z_{K}}\left|\bar{K}^{0}\right\rangle, \\
\left|K_{S}^{\text {in }}\right\rangle & =p_{K} \sqrt{1-z_{K}}\left|K^{0}\right\rangle+q_{K} \sqrt{1+z_{K}}\left|\bar{K}^{0}\right\rangle, \\
\left\langle K_{L}^{\text {out }}\right| & =\frac{1}{2 p_{K} q_{K}}\left(q_{K} \sqrt{1+z_{K}}\left\langle K^{0}\right|-p_{K} \sqrt{1-z_{K}}\left\langle\bar{K}^{0}\right|\right), \\
\left\langle K_{S}^{\text {out }}\right| & =\frac{1}{2 p_{K} q_{K}}\left(q_{K} \sqrt{1-z_{K}}\left\langle K^{0}\right|+p_{K} \sqrt{1+z_{K}}\left\langle\bar{K}^{0}\right|\right) .
\end{aligned}
$$

Since these mass eigenstates in $\mathrm{K}$ meson system are shown in flavor states, we specifically can calculate the amplitudes of transition from incoming states B meson to outgoing 
states $\psi K_{L, S}$

$$
\begin{aligned}
& A_{\psi K_{S}}=\left\langle\psi K_{S}^{\text {out }} \mid B_{0}^{\text {in }}\right\rangle=\frac{1}{2 p_{K} q_{K}}\left(q_{K} \sqrt{1-z_{K}} A_{\psi K^{0}}+p_{K} \sqrt{1+z_{K}} A_{\psi \bar{K}^{0}}\right), \\
& A_{\psi K_{L}}=\left\langle\psi K_{L}^{\text {out }} \mid B_{0}^{\text {in }}\right\rangle=\frac{1}{2 p_{K} q_{K}}\left(q_{K} \sqrt{1+z_{K}} A_{\psi K^{0}}-p_{K} \sqrt{1-z_{K}} A_{\psi \bar{K}^{0}}\right), \\
& \bar{A}_{\psi K_{S}}=\left\langle\psi K_{S}^{\text {out }} \mid \bar{B}_{0}^{\text {in }}\right\rangle=\frac{1}{2 p_{K} q_{K}}\left(q_{K} \sqrt{1-z_{K}} \bar{A}_{\psi K^{0}}+p_{K} \sqrt{1+z_{K}} \bar{A}_{\psi \bar{K}^{0}}\right), \\
& \bar{A}_{\psi K_{L}}=\left\langle\psi K_{L}^{\text {out }} \mid \bar{B}_{0}^{\text {in }}\right\rangle=\frac{1}{2 p_{K} q_{K}}\left(q_{K} \sqrt{1+z_{K}} \bar{A}_{\psi K^{0}}-p_{K} \sqrt{1-z_{K}} \bar{A}_{\psi \bar{K}^{0}}\right) .
\end{aligned}
$$

Hence, we can obtain eqs. (3.11), (3.22), (3.23) and (3.24). We also can write down inverse decay amplitudes of eqs. (B.12)-(B.15)

$$
\begin{aligned}
& A_{\psi K_{S}}^{\mathrm{ID}}=\left\langle B_{0}^{\text {out }} \mid \psi K_{S}^{\mathrm{in}}\right\rangle=\left(p_{K} \sqrt{1-z_{K}} A_{\psi K^{0}}^{\mathrm{ID}}+q_{K} \sqrt{1+z_{K}} A_{\psi \bar{K}^{0}}^{\mathrm{ID}}\right), \\
& A_{\psi K_{L}}^{\mathrm{ID}}=\left\langle B_{0}^{\mathrm{out}} \mid \psi K_{L}^{\mathrm{in}}\right\rangle=\left(p_{K} \sqrt{1+z_{K}} A_{\psi K^{0}}^{\mathrm{ID}}-q_{K} \sqrt{1-z_{K}} A_{\psi \bar{K}^{0}}^{\mathrm{ID}}\right), \\
& \bar{A}_{\psi K_{S}}^{\mathrm{ID}}=\left\langle\bar{B}_{0}^{\mathrm{out}} \mid \psi K_{S}^{\mathrm{in}}\right\rangle=\left(p_{K} \sqrt{1-z_{K}} \bar{A}_{\psi K^{0}}^{\mathrm{ID}}+q_{K} \sqrt{1+z_{K}} \bar{A}_{\psi \bar{K}^{0}}^{\mathrm{ID}}\right), \\
& \bar{A}_{\psi K_{L}}^{\mathrm{ID}}=\left\langle\bar{B}_{0}^{\text {out }} \mid \psi K_{L}^{\mathrm{in}}\right\rangle=\left(p_{K} \sqrt{1+z_{K}} \bar{A}_{\psi K^{0}}^{\mathrm{ID}}-q_{K} \sqrt{1-z_{K}} \bar{A}_{\psi \bar{K}^{0}}^{\mathrm{ID}}\right) .
\end{aligned}
$$

\section{List of coefficients of time dependent decay rates for process (I)}

In this appendix, we show the coefficients of the time dependent decay rates in eq. (2.1) which are needed for calculation of the asymmetry of process (I).

$$
\begin{aligned}
& S_{\left(\psi K_{L}\right) \perp, l^{-} X}=S_{\psi K_{L}}-S_{\psi K_{L}} z^{R}-z^{I}-G_{\psi K_{L}} \theta_{\psi K_{L}}^{I}, \\
& S_{\left(l^{+} X\right) \perp, \psi K_{S}}=S_{\psi K_{S}}+S_{\psi K_{S}} z^{R}-z^{I}-G_{\psi K_{S}} \theta_{\psi K_{S}}^{I}, \\
& C_{\left(\psi K_{L}\right) \perp, l^{-} X}=C_{\psi K_{L}}-S_{\psi K_{L}} S_{l^{-}}+G_{\psi K_{L}} z^{R}+S_{\psi K_{L}} z^{I}+\theta_{\psi K_{L}}^{R}, \\
& C_{\left(l^{+} X\right) \perp, \psi K_{S}}=-C_{\psi K_{S}}-S_{\psi K_{S}} S_{l^{+}}-G_{\psi K_{S}} z^{R}+S_{\psi K_{S}} z^{I}-\theta_{\psi K_{S}}^{R}, \\
& \kappa_{\left(\psi K_{L}\right) \perp, l^{-} X}=1-G_{\psi K_{L}} G_{l^{-}}-\left(G_{\psi K_{L}}+1\right) z^{R}-S_{\psi K_{L}} z^{I}, \\
& \kappa_{\left(l^{+} X\right) \perp, \psi K_{S}}=1-G_{\psi K_{S}} G_{l^{+}}+\left(G_{\psi K_{S}}+1\right) z^{R}-S_{\psi K_{S}} z^{I}, \\
& \sigma_{\left(\psi K_{L}\right) \perp, l^{-} X}=G_{l^{-}}-G_{\psi K_{L}}+\left(1+G_{\psi K_{L}}\right) z^{R}-S_{\psi K_{L}} \theta_{\psi K_{L}}^{I}, \\
& \sigma_{\left(l^{+} X\right) \perp \psi K_{S}}=G_{\psi K_{S}}-G_{l^{+}}+\left(1+G_{\psi K_{S}}\right) z^{R}+S_{\psi K_{S}} \theta_{\psi K_{S}}^{I}, \\
& \frac{S_{\left(\psi K_{L}\right) \perp, l^{-} X}}{\kappa_{\left(\psi K_{L}\right) \perp, l^{-} X}}=S_{\psi K_{L}}+S_{\psi K_{L}} G_{\psi K_{L}} G_{l^{-}}+S_{\psi K_{L}} G_{\psi K_{L}} z^{R}+\left(S_{\psi K_{L}}^{2}-1\right) z^{I}-G_{\psi K_{L}} \theta_{\psi K_{L}}^{I}, \\
& \frac{S_{\left(l^{+} X\right) \perp, \psi K_{S}}}{\kappa_{\left(l^{+} X\right) \perp, \psi K_{S}}}=S_{\psi K_{S}}+S_{\psi K_{S}} G_{\psi K_{S}} G_{l^{+}}-S_{\psi K_{S}} G_{\psi K_{S}} z^{R}+\left(S_{\psi K_{S}}^{2}-1\right) z^{I}-G_{\psi K_{S}} \theta_{\psi K_{S}}^{I},
\end{aligned}
$$




$$
\begin{aligned}
& \frac{C_{\left(\psi K_{L}\right) \perp, l^{-} X}}{\kappa_{\left(\psi K_{L}\right) \perp, l^{-} X}} \simeq C_{\left(\psi K_{L}\right) \perp, l^{-} X}, \\
& \frac{C_{\left(l^{+} X\right) \perp, \psi K_{S}}}{\kappa_{(l+X) \perp, \psi K_{S}}} \simeq C_{(l+X) \perp, \psi K_{S}}, \\
& \frac{\sigma_{\left(\psi K_{L}\right) \perp, l^{-} X}}{\kappa_{\left(\psi K_{L}\right) \perp, l^{-} X}} \simeq-G_{\psi K_{L}}, \\
& \frac{\sigma_{\left(l^{+} X\right) \perp, \psi K_{S}}}{\kappa_{\left(l^{+} X\right) \perp, \psi K_{S}}} \simeq G_{\psi K_{S}},
\end{aligned}
$$

where we keep only the leading term for $\frac{\sigma}{\kappa}$, since it will be multiplied by a small number $y$ in the formulae of the decay rate eq. (2.1).

\section{Expressions for $N_{R}, \Delta \mathcal{S}, \Delta \mathcal{C}, \Delta \sigma, \hat{\sigma}, \hat{\mathcal{S}}$ and $\hat{\mathcal{C}}$}

The quantity $N_{R}$, defined in eq. (2.4), denotes the ratio of a normalization for rates. Since we compute the asymmetry including the effect of $N_{R}$, its expression should be clarified. In this appendix section, we calculate $N_{R}$, and obtain the expressions of parameters as $\Delta \mathcal{S}, \Delta \mathcal{C}, \Delta \sigma, \hat{\sigma}, \hat{\mathcal{S}}$ and $\hat{\mathcal{C}}$ for the process (I). In deriving formulae, we use eqs. (C.1)-(C.14). Expanding $N_{R}$ with respect to small parameters, we obtain the general structure of $N_{R}$ at first order approximation.

$$
\begin{aligned}
N_{R} & =\frac{N_{(3) \perp, 4}}{N_{(1) \perp, 2}} \frac{\kappa_{(3) \perp, 4}}{\kappa_{(1) \perp, 2}} \\
& =\frac{\mathcal{N}_{3} \mathcal{N}_{4}\left[1+\left(C_{3}+C_{4}\right)\left(R_{M}-z^{R}\right)\right]}{\mathcal{N}_{1} \mathcal{N}_{2}\left[1+\left(C_{1}+C_{2}\right)\left(R_{M}-z^{R}\right)\right]} \frac{\kappa_{(3) \perp, 4}^{l}\left(1+\frac{\Delta \kappa_{(3) \perp, 4}}{\kappa_{(3) \perp, 4}^{l}}\right)}{\kappa_{(1) \perp, 2}^{l}\left(1+\frac{\Delta \kappa_{(1) \perp, 2}}{\kappa_{(1) \perp, 2}^{l}}\right)} \\
& \simeq \frac{\mathcal{N}_{3} \mathcal{N}_{4}}{\mathcal{N}_{1} \mathcal{N}_{2}} \frac{\kappa_{(3) \perp, 4}^{l}}{\kappa_{(1) \perp, 2}^{l}}\left[1+\left(C_{3}+C_{4}-C_{1}-C_{2}\right)\left(R_{M}-z^{R}\right)+\frac{\Delta \kappa_{(3) \perp, 4}}{\kappa_{(3) \perp, 4}^{l}}-\frac{\Delta \kappa_{(1) \perp, 2}}{\kappa_{(1) \perp, 2}^{l}}\right],
\end{aligned}
$$

where superscript ${ }^{l}$ expresses the leading part and $\Delta$ expresses the small part such as, $\kappa_{(1) \perp, 2}=\kappa_{(1) \perp, 2}^{l}+\Delta \kappa_{(1) \perp, 2}$ and $\kappa_{(3) \perp, 4}=\kappa_{(3) \perp, 4}^{l}+\Delta \kappa_{(3) \perp, 4}$. For the processes given in eq. (4.1), $\kappa_{(1) \perp, 2}^{l}=\kappa_{(3) \perp, 4}^{l}=1\left(f_{1}=\psi K_{L}, f_{2}=l^{-} X, f_{3}=l^{+} X, f_{4}=\psi K_{S}\right.$ for process (I) $)$ is satisfied and $N_{R}^{I}$ is written as,

$$
\begin{aligned}
N_{R}^{I}= & \frac{\mathcal{N}_{l^{+} X} \mathcal{N}_{\psi K_{S}}}{\mathcal{N}_{\psi K_{L}} \mathcal{N}_{l^{-} X}}\left[1+\left(C_{\psi K_{S}}-C_{\psi K_{L}}+C_{l^{+}}-C_{l^{-}}\right)\left(R_{M}-z^{R}\right)\right. \\
& \left.+\Delta \kappa_{\left(l^{+} X\right) \perp, \psi K_{S}}-\Delta \kappa_{\left(\psi K_{L}\right) \perp, l^{-} X}\right] \\
\simeq & \frac{\mathcal{N}_{\psi K_{S}} \mathcal{N}_{l^{+} X}}{\mathcal{N}_{\psi K_{L}} \mathcal{N}_{l^{-} X}}\left[1+2\left(R_{M}-z^{R}\right)+2\left(z^{R}-S z^{I}-G \hat{\lambda}_{l}^{R}\right)\right] \\
= & \frac{\mathcal{N}_{\psi K_{S}} \mathcal{N}_{l^{+} X}}{\mathcal{N}_{\psi K_{L}} \mathcal{N}_{l^{-} X}}\left[1+2\left(-S z^{I}+R_{M}-G \hat{\lambda}_{l}^{R}\right)\right] .
\end{aligned}
$$


Deviation of $\mathcal{N}_{l^{+} X} / \mathcal{N}_{l^{-} X}$ and $\mathcal{N}_{\psi K_{S}} / \mathcal{N}_{\psi K_{L}}$ from 1 is written in terms of small parameters as,

$$
\begin{aligned}
\frac{\mathcal{N}_{l^{+} X}}{\mathcal{N}_{l^{-} X}} & =1-2\left(C_{\xi}^{l}+\xi_{l}^{R}\right), \quad \frac{\mathcal{N}_{\psi K_{S}}}{\mathcal{N}_{\psi K_{L}}}=1+2 \hat{\lambda}_{\mathrm{wst}}^{R} . \\
N_{R}^{I} & =1+\Delta N_{R}^{I}=1+2\left[-S z^{I}+R_{M}+\hat{\lambda}_{\mathrm{wst}}^{R}-G \hat{\lambda}_{l}^{R}-C_{\xi}^{l}-\xi_{l}^{R}\right] .
\end{aligned}
$$

Note that $\Delta N_{R}^{I}$ is a small number.

We can also write down the expressions of $\Delta S^{I}$ and $\Delta C^{I}$.

$$
\begin{aligned}
\Delta \mathcal{S}^{I} & =\left(\frac{\mathcal{S}_{\left(\psi K_{L}\right) \perp, l^{-} X}}{\kappa_{\left(\psi K_{L}\right) \perp, l^{-} X}}-\frac{\mathcal{S}_{\left(l^{+} X\right) \perp, \psi K_{S}}}{\kappa_{\left(l^{+} X\right) \perp, \psi K_{S}}}\right)-\frac{\Delta N_{R}^{I}}{2}\left(\frac{\mathcal{S}_{\left(\psi K_{L}\right) \perp, l^{-} X}}{\kappa_{\left(\psi K_{L}\right) \perp, l^{-} X}}+\frac{\mathcal{S}_{\left(l^{+} X\right) \perp, \psi K_{S}}}{\kappa_{\left(l^{+} X\right) \perp, \psi K_{S}}}\right) \\
& \simeq \frac{\mathcal{S}_{\left(\psi K_{L}\right) \perp, l^{-} X}}{\kappa_{\left(\psi K_{L}\right) \perp, l^{-} X}}-\frac{\mathcal{S}_{\left(l^{+} X\right) \perp, \psi K_{S}}}{\kappa_{\left(l^{+} X\right) \perp, \psi K_{S}}}=-2\left[S\left(1-G z^{R}\right)-G \theta_{K}^{I}+G S \Delta \lambda_{l}^{R}\right], \\
\Delta \mathcal{C}^{I} & \simeq \frac{\mathcal{C}_{\left(\psi K_{L}\right) \perp, l^{-} X}}{\kappa_{\left(\psi K_{L}\right) \perp, l^{-} X}}-\frac{\mathcal{C}_{\left(l^{+} X\right) \perp, \psi K_{S}}}{\kappa_{\left(l^{+} X\right) \perp, \psi K_{S}}}=2\left[C-S z^{I}+\theta_{K}^{R}+S \Delta \lambda_{l}^{I}\right] .
\end{aligned}
$$

We calculate only the leading part of $\Delta \sigma^{I}$ and $\hat{\sigma}^{I}$, since the sub-leading part of $\Delta \sigma^{I}$ and $\hat{\sigma}^{I}$ is suppressed when multiplied with $y \Gamma t$.

$$
\Delta \sigma^{I l}=0, \quad \hat{\sigma}^{I l}=2 G .
$$

We write down the expressions for $\hat{\mathcal{S}}^{I}$ and $\hat{\mathcal{C}}^{I}$ as follows,

$$
\begin{aligned}
\hat{\mathcal{S}}^{I} & =\left(\frac{\mathcal{S}_{\left(\psi K_{L}\right) \perp, l^{-} X}}{\kappa_{\left(\psi K_{L}\right) \perp, l^{-} X}}+\frac{\mathcal{S}_{\left(l^{+} X\right) \perp, \psi K_{S}}}{\kappa_{\left(l^{+} X\right) \perp, \psi K_{S}}}\right)-\frac{\Delta N_{R}^{I}}{2}\left(\frac{\mathcal{S}_{\left(\psi K_{L}\right) \perp, l^{-} X}}{\kappa_{\left(\psi K_{L}\right) \perp, l^{-} X}}-\frac{\mathcal{S}_{\left(l^{+} X\right) \perp, \psi K_{S}}}{\kappa_{\left(l^{+} X\right) \perp, \psi K_{S}}}\right) \\
& \simeq 2 G\left(z_{K}^{I}-\Delta \lambda_{\mathrm{wst}}^{I}\right)+2\left(S^{2}-1\right) z^{I}+2 G S \hat{\lambda}_{l}^{R}+S \Delta N_{R}^{I} \\
& =2\left[G\left(z_{K}^{I}-\Delta \lambda_{\mathrm{wst}}^{I}\right)-z^{I}+S R_{M}+S \hat{\lambda}_{\mathrm{wst}}^{R}-S C_{\xi}^{l}-S \xi_{l}^{R}\right], \\
\hat{\mathcal{C}}^{I} & \simeq \frac{\mathcal{C}_{\left(\psi K_{L}\right) \perp, l^{-} X}}{\kappa_{\left(\psi K_{L}\right) \perp, l^{-} X}}+\frac{\mathcal{C}_{\left(l^{+} X\right) \perp, \psi K_{S}}}{\kappa_{\left(l^{+} X\right) \perp, \psi K_{S}}}=2\left[z_{K}^{R}-\Delta \lambda_{\mathrm{wst}}^{R}-G z^{R}-S \hat{\lambda}_{l}^{I}\right] .
\end{aligned}
$$

\section{E The relation among coefficients of the asymmetries for processes (I)- (IV)}

In this appendix, we show the relation among the coefficients for different processes (I-IV). First, we note the coefficients of the process II(IV) are obtained by changing the sign of the mixing parameter $q_{K}$ and $z_{K}$ of I(III). The change of the sign of $q_{K}$ leads to the change of the sign for $S, G$ and $\lambda_{\text {wst. }}$. Next, we show a simple rule which enables one to obtain the coefficients for table 5 , with the coefficients of table 7 . For this purpose we do not 
substitute \pm 1 for $C_{l^{ \pm}}$respectively and write the coefficients of asymmetry for process IV,

$$
\begin{aligned}
R_{T}^{I V}= & -S z^{I}+\frac{1}{2}\left(C_{l^{+}}-C_{l^{-}}\right) R_{M}-\xi_{l}^{R}-C_{\xi}^{l}+\hat{\lambda}_{\mathrm{wst}}^{R}-G \hat{\lambda}_{l}^{R}, \\
C_{T}^{I V}= & \frac{1}{2}\left(C_{l^{-}}-C_{l^{+}}\right) C+S z^{I}+\frac{1}{2}\left(C_{l^{-}} \theta_{K_{L}}^{R}-C_{l^{+}} \theta_{K_{S}}^{R}\right)-S \Delta \lambda_{l}^{I}, \\
S_{T}^{I V}= & \frac{1}{2}\left(C_{l^{-}}-C_{l^{+}}\right) S+S G z^{R}+\frac{G}{2}\left(C_{l^{+}} \theta_{K_{S}}^{I}-C_{l^{-}} \theta_{K_{L}}^{I}\right) \\
& -G S\left(C_{l^{+}} \operatorname{Re}\left[\lambda_{l^{+}}\right]+C_{l^{-}} \operatorname{Re}\left[\lambda_{l^{-}}^{-1}\right]\right), \\
B_{T}^{I V}= & S\left[-G z_{K}^{I}+z^{I}+\frac{C_{l^{-}}-C_{l^{+}}}{2} S R_{M}+S \xi_{l}^{R}\right]+S^{2} C_{\xi}^{l}-S^{2} \hat{\lambda}_{\mathrm{wst}}^{R}+S G \Delta \lambda_{\mathrm{wst}}^{I}, \\
D_{T}^{I V}= & S\left[z_{K}^{R}-G z^{R}\right]-S \Delta \lambda_{\mathrm{wst}}^{R}+\frac{C_{l^{-}}-C_{l^{+}}}{2} S^{2} \hat{\lambda}_{l}^{I}, \\
E_{T}^{I V}= & \frac{C_{l^{-}}-C_{l^{+}}}{2} G S .
\end{aligned}
$$

When $l^{+}$and $l^{-}$in eq. (D.3) are exchanged, the sign of $C_{\xi}^{l}$ and $\xi_{l}^{R}$ is reversed. According to eqs. (3.40)-(3.41), the sign of $\hat{\lambda}_{l}^{I}$ and $\Delta \lambda_{l}^{R}$ also changes. Additionally, one needs to interchange $C_{l^{+}}$and $C_{l^{-}}$in eqs. (E.1)-(E.7) and one can obtain the coefficients of asymmetry for process II.

\section{F Calculation of equivalence conditions}

In this appendix, we give the derivation of eqs. (5.3)-(5.4). The expression of the final state of signal side in figure (2) is,

$$
\left|B_{\left(\rightarrow \psi K_{S}\right) \perp}\right\rangle=N_{\left(\rightarrow \psi K_{S}\right) \perp}\left(\bar{A}_{\psi K_{S}}\left|B^{0}\right\rangle-A_{\psi K_{S}}\left|\bar{B}^{0}\right\rangle\right)
$$

since the state is orthogonal to $\left\langle\psi K_{S}\right|$. The state orthogonal to $\left|\psi K_{L}\right\rangle$ is

$$
\left\langle B_{\left(\psi K_{L} \rightarrow\right) \perp}\right|=N_{\left(\psi K_{L} \rightarrow\right) \perp}\left(\bar{A}_{\psi K_{L}}^{\mathrm{ID}}\left\langle B^{0}\right|-A_{\psi K_{L}}^{\mathrm{ID}}\left\langle\bar{B}^{0}\right|\right) .
$$

Similarly, one can write down,

$$
\begin{aligned}
\left\langle B_{\left(\psi K_{S} \rightarrow\right) \perp}\right| & =N_{\left(\psi K_{S} \rightarrow\right) \perp}\left(\bar{A}_{\psi K_{S}}^{\mathrm{ID}}\left\langle B^{0}\right|-A_{\psi K_{S}}^{\mathrm{ID}}\left\langle\bar{B}^{0}\right|\right), \\
\left|B_{\left(\rightarrow \psi K_{L}\right) \perp}\right\rangle & =N_{\left(\rightarrow \psi K_{L}\right) \perp}\left(\bar{A}_{\psi K_{L}}\left|B^{0}\right\rangle-A_{\psi K_{L}}\left|\bar{B}^{0}\right\rangle\right) .
\end{aligned}
$$

Calculating the inner product of eqs. (F.1) and (F.2), we obtain

$$
\begin{aligned}
\left\langle B_{\left(\psi K_{L} \rightarrow\right) \perp} \mid B_{\left(\rightarrow \psi K_{S}\right) \perp}\right\rangle= & N_{\left(\rightarrow \psi K_{S}\right) \perp} N_{\left(\psi K_{L} \rightarrow\right) \perp}\left(\bar{A}_{\psi K_{S}} \bar{A}_{\psi K_{L}}^{\mathrm{ID}}+A_{\psi K_{S}} A_{\psi K_{L}}^{\mathrm{ID}}\right) \\
= & \frac{1}{2} N_{\left(\rightarrow \psi K_{S}\right) \perp} N_{\left(\psi K_{L} \rightarrow\right) \perp}\left[A_{\psi K^{0}} A_{\psi K^{0}}^{\mathrm{ID}}-\bar{A}_{\psi \bar{K}^{0}} \bar{A}_{\psi \bar{K}^{0}}^{\mathrm{ID}}\right. \\
& \left.-\frac{q_{K}}{p_{K}}\left(A_{\psi K^{0}} A_{\psi \bar{K}^{0}}^{\mathrm{ID}}+\bar{A}_{\psi \bar{K}^{0}}^{\mathrm{ID}} \bar{A}_{\psi K^{0}}\right)+\frac{p_{K}}{q_{K}}\left(A_{\psi \bar{K}^{0}} A_{\psi K^{0}}^{\mathrm{ID}}+\bar{A}_{\psi \bar{K}^{0}} \bar{A}_{\psi K^{0}}^{\mathrm{ID}}\right)\right] \\
= & \frac{N_{\left(\rightarrow \psi K_{S}\right) \perp} N_{\left(\psi K_{L} \rightarrow\right) \perp}}{2}\left(A_{\psi K^{0}} A_{\psi K^{0}}^{\mathrm{ID}}+\bar{A}_{\psi \bar{K}^{0}} \bar{A}_{\psi \bar{K}^{0}}^{\mathrm{ID}}\right)\left[\theta_{K}+\Delta \lambda_{\text {wst }}\right],
\end{aligned}
$$


where we used eqs. (B.12)-(B.19). The inner product in eq. (F.5) was previously obtained in [6]. In eq. (F.5), we compute it with our notation including the normalization constant and have ignored the second order of small parameters $z_{K}, \theta_{\psi K^{0}}, \bar{\theta}_{\psi \bar{K}^{0}}, \hat{\lambda}_{\mathrm{wst}}$ and $\Delta \lambda_{\mathrm{wst}}$.

Next, we show the derivation of the first line of eq. (5.3) and the second line of eq. (5.4). The states are given as,

$$
\begin{aligned}
& \left\langle B_{\left(l^{-} X \rightarrow\right) \perp}\right|=N_{\left(l^{-} \rightarrow\right) \perp}\left(\bar{A}_{l^{-}}^{\mathrm{ID}}\left\langle B^{0}\right|-A_{l^{-}}^{\mathrm{ID}}\left\langle\bar{B}^{0}\right|\right), \\
& \left|B_{\left(\rightarrow l^{+} X\right) \perp}\right\rangle=N_{\left(\rightarrow l^{+}\right) \perp}\left(\bar{A}_{l^{+}}\left|B^{0}\right\rangle-A_{l^{+}}\left|\bar{B}^{0}\right\rangle\right), \\
& \left\langle B_{\left(l^{+} X \rightarrow\right) \perp}=N_{\left(l^{+} \rightarrow\right) \perp}\left(\bar{A}_{l^{+}}^{\mathrm{ID}}\left\langle B^{0}\right|-A_{l^{+}}^{\mathrm{ID}}\left\langle\bar{B}^{0}\right|\right),\right. \\
& \left|B_{\left(\rightarrow l^{-} X\right) \perp}\right\rangle=N_{\left(\rightarrow l^{-}\right) \perp}\left(\bar{A}_{l^{-}}\left|B^{0}\right\rangle-A_{l^{-}}\left|\bar{B}^{0}\right\rangle\right) .
\end{aligned}
$$

Their inner product is,

$$
\begin{aligned}
\left\langle B_{\left(l^{-} X \rightarrow\right) \perp} \mid B_{\left(\rightarrow l^{+} X\right) \perp}\right\rangle & =N_{\left(l^{-} \rightarrow\right) \perp} N_{\left(\rightarrow l^{+}\right) \perp}\left(\bar{A}_{l^{-}}^{\mathrm{ID}} \bar{A}_{l^{+}}+A_{l^{-}}^{\mathrm{ID}} A_{l^{+}}\right) \\
& =2 N_{\left(l^{-} \rightarrow\right) \perp} N_{\left(\rightarrow l^{+}\right) \perp} A_{l^{+}} \bar{A}_{l^{-}}^{\mathrm{ID}} \frac{p}{q} \lambda_{l^{+}} .
\end{aligned}
$$

The proportionality to the wrong sign decay amplitude $\lambda_{l^{+}}$is derived in [6].

\section{Acknowledgments}

We would like to thank Dr. T. Shindou for useful comments and suggestion, and helpful correspondence by A. Efrati, Y. Nir and Y. Soreq.

Open Access. This article is distributed under the terms of the Creative Commons Attribution License (CC-BY 4.0), which permits any use, distribution and reproduction in any medium, provided the original author(s) and source are credited.

\section{References}

[1] M.C. Banuls and J. Bernabeu, CP, T and CPT versus temporal asymmetries for entangled states of the $B_{d}$-system, Phys. Lett. B 464 (1999) 117 [hep-ph/9908353] [INSPIRE].

[2] E. Alvarez and A. Szynkman, Direct test of time reversal invariance violation in B mesons, Mod. Phys. Lett. A 23 (2008) 2085 [hep-ph/0611370] [INSPIRE].

[3] J. Bernabeu, F. Martinez-Vidal and P. Villanueva-Perez, Time Reversal Violation from the entangled $B^{0} \bar{B}^{0}$ system, JHEP 08 (2012) 064 [arXiv:1203.0171] [INSPIRE].

[4] BABAR collaboration, J.P. Lees et al., Observation of Time Reversal Violation in the $B^{0}$ Meson System, Phys. Rev. Lett. 109 (2012) 211801 [arXiv:1207.5832] [INSPIRE].

[5] K.R. Schubert, T Violation and CPT Tests in Neutral-Meson Systems, arXiv:1409.5998 [INSPIRE].

[6] E. Applebaum, A. Efrati, Y. Grossman, Y. Nir and Y. Soreq, Subtleties in the BABAR measurement of time-reversal violation, Phys. Rev. D 89 (2014) 076011 [arXiv:1312.4164] [INSPIRE].

[7] Y. Grossman, A.L. Kagan and Z. Ligeti, Can the $C P$ asymmetries in $B \rightarrow \psi K(s)$ and $B \rightarrow \psi K(L)$ differ?, Phys. Lett. B 538 (2002) 327 [hep-ph/0204212] [INSPIRE]. 
[8] A. Lenz and U. Nierste, Numerical Updates of Lifetimes and Mixing Parameters of B Mesons, arXiv:1102.4274 [INSPIRE].

[9] I.I. Bigi and A.I. Sanda, CP violation, Camb. Monogr. Part. Phys. Nucl. Phys. Cosmol. 9 (2000) 1 [INSPIRE].

[10] H.-n. Li and S. Mishima, Penguin pollution in the $B^{0} \rightarrow J / \psi K(S)$ decay, JHEP 03 (2007) 009 [hep-ph/0610120] [INSPIRE].

[11] A.S. Dighe, T. Hurth, C.S. Kim and T. Yoshikawa, Measurement of the lifetime difference of $B_{d}$ mesons: possible and worthwhile?, Nucl. Phys. B 624 (2002) 377 [hep-ph/0109088] [INSPIRE].

[12] A. Dighe, T. Hurth, C.S. Kim and T. Yoshikawa, The Width difference of $B_{d}$ mesons, PoS (HEP2001) 096 [hep-ph/0112067] [INSPIRE].

[13] R.G. Sachs, Methods for Testing the CPT Theorem, Phys. Rev. 129 (1963) 2280 [InSPIRE].

[14] C.P. Enz and R.R. Lewis, On the phenomenological description of CP-violation for $K$ mesons and its consequences, Helv. Phys. Acta 38 (1965) 860 [INSPIRE].

[15] L. Álvarez-Gaumé, C. Kounnas, S. Lola and P. Pavlopoulos, Violation of time reversal invariance and CPLEAR measurements, Phys. Lett. B 458 (1999) 347 [hep-ph/9812326] [INSPIRE].

[16] M. Beuthe, G. Lopez Castro and J. Pestieau, Field theory approach to $K^{0}-\overline{K^{0}}$ and $B^{0}-\overline{B^{0}}$ systems, Int. J. Mod. Phys. A 13 (1998) 3587 [hep-ph/9707369] [InSPIRE].

[17] J.P. Silva, On the use of the reciprocal basis in neutral meson mixing, Phys. Rev. D 62 (2000) 116008 [hep-ph/0007075] [InSPIRE]. 